\title{
Iguales en las diferencias: iniciativas de investigación transnacionales sobre Informática Educativa en Latinoamérica en el periodo 2010-2020
}

\author{
Title: Equal in differences: transnational research initiatives on Computers in Education in \\ Latin America during the 2010-2020 period
}

Ismar Frango Silveira

U. Presbiteriana Mackenzie

ismar.silveira@mackenzie.br

Antonio Silva Sprock

Universidad Central de Venezuela

antonio.silva@ciens.ucv.ve

Jaime Muñoz-Arteaga

U. Autónoma de Aguascalientes

jaime.munoz@edu.uaa.mx

Regina Motz

Universidad de la República

rmotz@fing.edu.uy

\author{
Ana Casali \\ Universidad Nacional de Rosario \\ acasali@fceia.unr.edu.ar
}

Cesar Alberto Collazos

Universidad del Cauca

ccollazo@unicauca.edu.co

Jorge Maldonado-Mahauad

Universidad de Cuenca

jorge.maldonado@ucuenca.edu.ec

Virginia Rodés Paragarino
Universidad de la República
virginia.rodes@cse.udelar.edu.uy
Ana Verónica Morales Bezeira

Universidad Central de Venezuela ana.morales@ciens.ucv.ve

\author{
Cristian Cechinel \\ U. Federal de Santa Catarina \\ cristian.cechinel@ufsc.br
}

\author{
Mario Chacón Rivas \\ TEC - Costa Rica \\ machacon@itcr.ac.cr \\ Xavier Ochoa \\ New York University \\ xavier.ochoa@nyu.edu
}

\section{Resumen}

Es sabido que América Latina es la región más desigual del planeta. Para hacer frente a esta realidad, una de las cuestiones clave es impulsar la colaboración en la región, que permita que la innovación y el conocimiento sean instrumentos fundamentales para erradicar la pobreza, combatir el hambre y mejorar la salud, así como para alcanzar un desarrollo sostenible, integrado, inclusivo y equitativo de la región. Este artículo presenta algunas iniciativas en el ámbito latinoamericano en el periodo 2010-2020, en la intersección entre la Computación y la Educación, que han surgido como redes colaborativas para fomentar la educación asistida por las Tecnologías de la Información y Comunicación. En ese sentido, se plantean desafios de investigación en el campo de la Informática Educativa y las perspectivas para nuevas colaboraciones posibles son delineadas.

Palabras-Clave: Latinoamérica, Desigualdad, Colaboración, Tecnologías Educativas.

\footnotetext{
Abstract

Latin America is known to be the most unequal region on the planet. To face reality, one of the key issues is to promote collaboration in the region, which allow innovation and knowledge to be fundamental instruments to eradicate poverty, combat hunger and improve health, as well as to achieve a sustainable, integrated, inclusive and equitable development of the region. This article presents some initiatives in the Latin American field in the 20102020 period, at the intersection between Computing and Education, which have emerged as collaborative networks to promote education supported by Information and Communication Technologies. In that sense, research challenges in the field of Educational Informatics are raised and the perspectives for new possible collaborations are outlined.

Keywords: Latin America, Inequalities, Collaboration, Computers in Education.

Cite as: Silveira, I. F., Casali, A., Morales Bezeira, A. V., Silva Sprock, A., Collazos, C. A., Cechinel, C., MuñozArteaga, J., Maldonado-Mahauad, J., Chacón-Rivas, M., Motz, R., Rodés-Paragarino, V., \& Ochoa, X. (2021). Iguales en las diferencias: iniciativas de investigación transnacionales sobre Informática Educativa en Latinoamérica en el periodo 2010-2020. Revista Brasileira de Informática na Educação, 29, 1060-1090. DOI: 10.5753/RBIE.2021.29.0.1060
} 


\section{Introducción}

Latinoamérica es ciertamente un crisol de culturas y realidades. Tiene una población estimada en 652 millones de habitantes, de los cuales alrededor de un 60\% es de hispanohablantes (los que tienen al Español como primera lengua) distribuidos en 19 países (Argentina, Bolivia, Chile, Colombia, Costa Rica, Cuba, Ecuador, El Salvador, Guatemala, Honduras, México, Nicaragua, Panamá, Paraguay, Perú, Puerto Rico, República Dominicana, Uruguay y Venezuela); un 30\% vive en Brasil y son lusohablantes; y un 10\% tienen lenguas autóctonas (Guaraní, Aymara, Nahuatl, Quechua, entre otras) o suelen adoptar el francés (como Haití y Guayana Francesa) y variantes de criollo como primera lengua. Pese a la casi-dicotomía lingüística en la cual se encuentra (la mayoría habla Español o Portugués), los distintos rasgos culturales, sociales y económicos y diferentes caminos históricos, los países de Latinoamérica guardan entre sí un importante conjunto de similitudes que hay que explorar, de manera sistémica, cuando uno habla de investigación con potencial de impacto social -en ese contexto se incluye toda la investigación en el campo de la Educación y más específicamente, lo que se refiere a la aplicación de las Tecnologías de la Información y Comunicación (TICs) en el campo educativo.

La Educación es un derecho humano fundamental (según la Declaración Universal de Derechos Humanos, disponible en https://es.unesco.org/themes/derecho-a-educacion, 1948). Este derecho es uno de los principios de la Agenda Mundial Educación 2030 y del Objetivo de Desarrollo Sostenible 4 (ODS 4) adoptado por la comunidad internacional. El ODS 4 está basado en los derechos humanos y tiene el propósito de garantizar el disfrute pleno del derecho a la educación como catalizador para lograr un desarrollo sostenible. Sin embargo, millones de niños y adultos siguen privados de oportunidades educativas, en muchos casos debido a factores sociales, culturales y económicos, en especial en la región Latinoamericana.

América Latina se encuentra entre las regiones con mayor nivel de desigualdad del mundo. De acuerdo al índice GINI ${ }^{1}$, los países de la región son en promedio $30 \%$ más desiguales que el resto del mundo. La distribución de índice de GINI (según las estimativas más recientes del Banco Mundial ${ }^{2}$ ) por países en Latinoamérica puede verse en la Figura 1. A continuación se muestran los datos de alcance de Internet en la población de cada país ${ }^{3}$ en la Figura 2 y un comparativo de algunos países en $\mathrm{PISA}^{4}$ (Programme for International Student Assessment) en la Figura 3. Esto conduce a una discusión sobre inequidad social, desigualdades en el acceso y permanencia en la educación.

\footnotetext{
${ }^{1}$ El coeficiente de GINI es el método más utilizado para medir la desigualdad salarial que existe entre los ciudadanos de un territorio, se computa entre 0 (máxima igualdad) y 1 (máxima desigualdad). El índice de GINI es el coeficiente de GINI expresado como un porcentaje, es decir, es el coeficiente de GINI multiplicado por 100.

${ }^{2}$ https://data.worldbank.org/indicator/SI.POV.GINI?locations=ZJ

${ }^{3} \mathrm{https}: / / \mathrm{www}$. internetworldstats.com/stats2.htm

${ }^{4}$ https://www.oecd.org/pisa/publications/pisa-2018-results.htm
} 


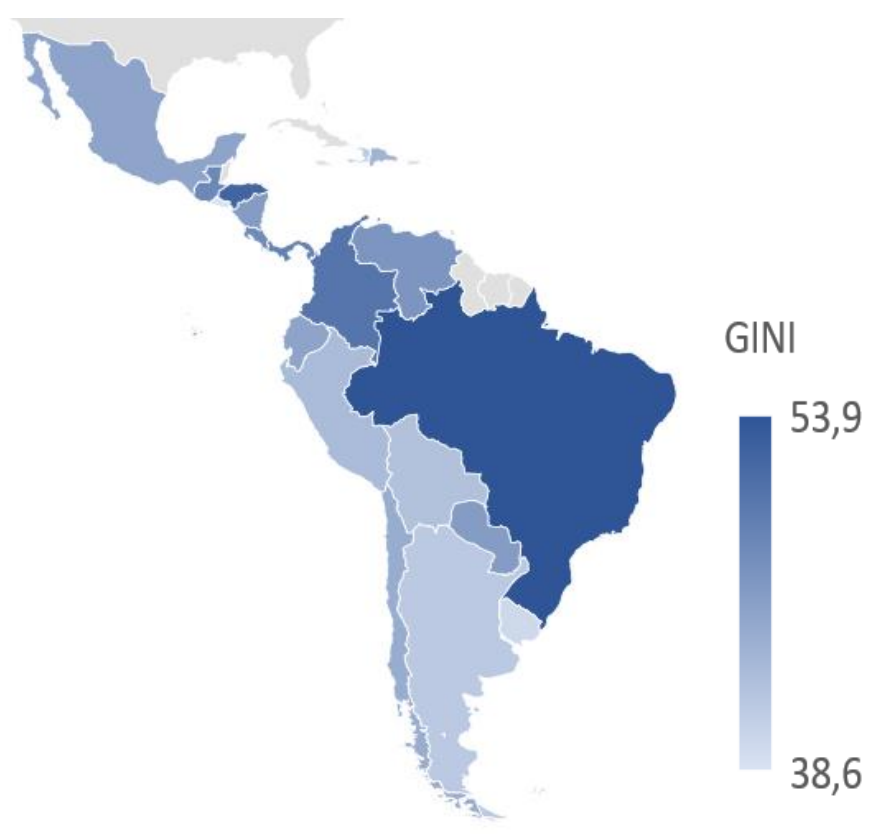

Figura 1: Índice de GINI (cuanto más grande, más inequidad). (Fuente: autores)

\section{GINI vs. Penetración de Internet}

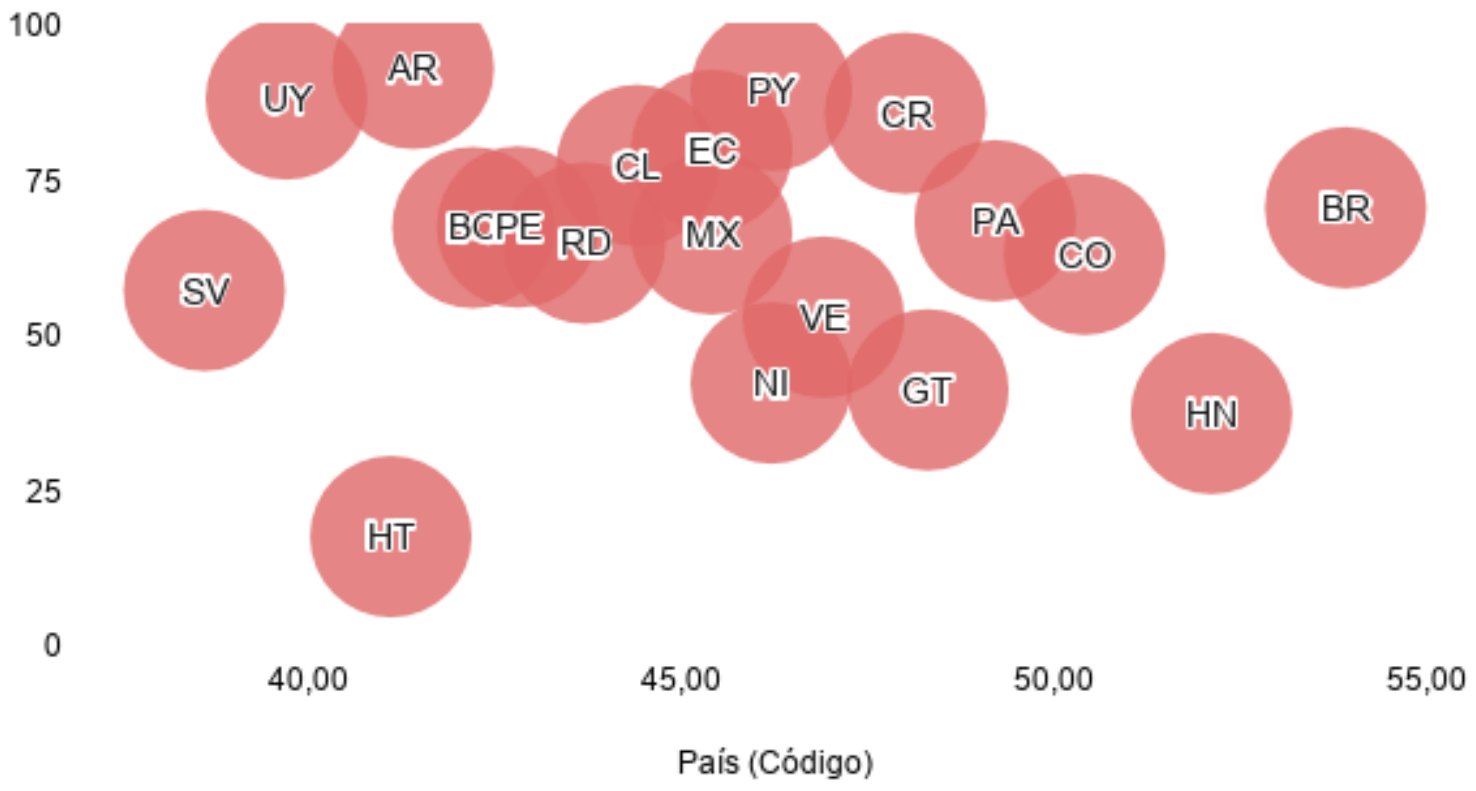

Figura 2: Índice de GINI (horizontal) vs Porcentaje de Conectividad a Internet (vertical) - los países son representados por sus códigos de Internet (Fuente: autores)

En la Figura 2, se puede verificar que hay países con índices bajos de inequidad, como Uruguay (UY) y Argentina (AR) y con un buen porcentaje de personas conectadas a la Internet. Hay países más pobres poseen índices de inequidad aún más pequeños y baja conectividad, como Haití (HT) y El Salvador (SV) - lo que refleja el impacto de las condiciones financieras al acceso a Internet. Brasil se destaca por tener el peor índice GINI de la región, aunque presenta una razonable conectividad.

Con relación al acceso a tecnologías y a Internet, hay una fuerte variabilidad según el país, observándose que alrededor del $64 \%$ de los latinoamericanos tienen algún acceso a internet, 
destacándose Argentina con cerca del 93\% de la población, seguida de Paraguay, Uruguay y Costa Rica, con más del $80 \%$ de población. Mientras tanto, menos del $20 \%$ de los habitantes de Haití poseen acceso a la Internet. La brecha de acceso a las TIC profundiza las desigualdades, condicionando el derecho a la salud, la educación y el trabajo. Como han mostrado Tomczyk et al. (2019), la variación de acceso se conecta a datos relacionados al estrato social, edad y localización.

La desigualdad en el acceso se observa en relación con el nivel de ingreso, $81 \%$ de los hogares del quintil de ingresos más alto (quinto) tiene conexión a Internet, mientras que los del primer y segundo quintil es del $38 \%$ y el $53 \%$ respectivamente. La desigualdad también se expresa entre los países: por ejemplo, en Brasil y Chile, más del 60\% de los hogares del primer quintil tiene conexión a Internet, mientras en Bolivia, Paraguay y Perú, sólo el 3\% la tiene, limitando las posibilidades de acceso al teletrabajo y la educación, lo cual se agrava en el marco de la emergencia debida al COVID-19.

Son importantes las diferencias en acceso a internet entre las áreas urbanas y rurales, ya que en Latinoamérica un promedio del $67 \%$ de los hogares urbanos está conectado a Internet, mientras que sólo lo está el $23 \%$ de los hogares rurales. Los grupos etarios con menos acceso a conectividad son los niños de 5 a 12 años y los adultos mayores de 65 años. Además, la situación de exclusión se profundiza con relación a las velocidades de conexión para el uso de soluciones digitales, siendo que, en el año 2020, en el 44\% de los países de la región no se alcanzaba la velocidad de descarga que permita realizar actividades en línea simultáneamente. Durante dicho año, más de 32 millones de niños que viven en hogares sin conexión no han podido acceder a las soluciones digitales para la continuidad educativa.

Las condiciones sociales vulnerables y los altos niveles de inequidad en muchos países de la región producen impactos negativos en la Educación. Menos del 1\% de los estudiantes latinoamericanos alcanzan puntajes de nivel alto en los exámenes PISA (OCDE PISA, 2015). La tasa de desvinculación escolar es alta, aunque se han destacado avances con un $92 \%$ que finaliza la etapa escolar, presentando, sin embargo, porcentajes bajos de finalización en algunos países como Brasil y México. Uno de cada cinco jóvenes de entre 15 y 24 años en América Latina no asiste a la escuela y no trabaja. La Figura 3 enseña los puntajes alcanzados en PISA 2018, junto al porcentaje de alumnos considerados con baja capacidad de lectura.

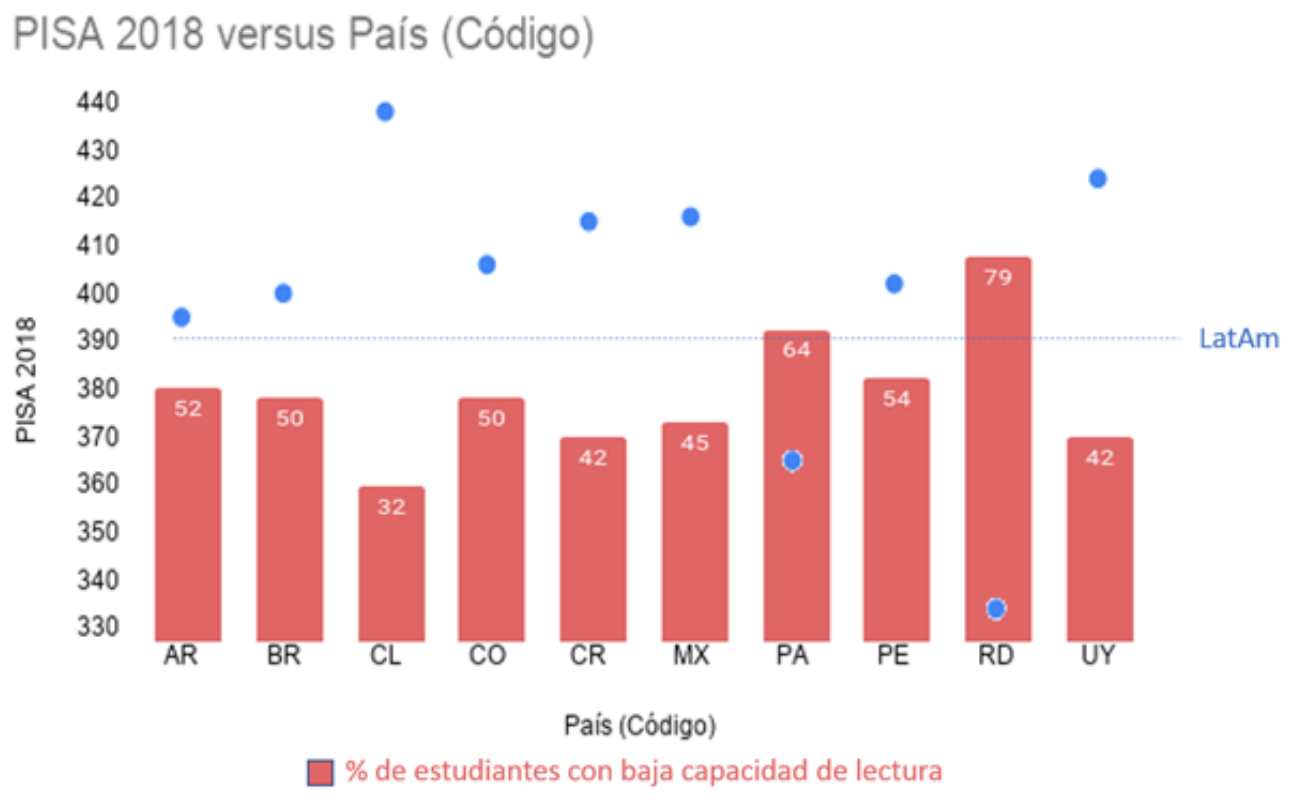

Figura 3. Desempeño de estudiantes de Latinoamérica en PISA 2018 (Fuente: autores) 
También en el caso de la Educación Superior (ES) son particularmente importantes los desafíos a los que se enfrenta la región, a partir de su progresiva digitalización. Este es un proceso transformador que impacta sustantivamente en todas las actividades de las Instituciones de ES (IES), permeando todos los procesos y ámbitos. Incluye el desarrollo de nuevas infraestructuras y el uso creciente de medios digitales y tecnologías para la enseñanza y el aprendizaje, la investigación, servicios de apoyo, administración y comunicación, como también la necesidad de que los estudiantes y el personal desarrollen nuevas habilidades digitales para el trabajo actual y futuro.

La última Conferencia Regional sobre Educación Superior en América Latina y el Caribe (CRES 2018) $)^{5}$ marca un mapa de ruta crítica, un plan de acción y un conjunto de principios y prioridades para que las IES de la región avancen en los Objetivos de Desarrollo Sostenible (ODS). Bajo el ODS 4, referido a educación y ES, en el centro de sus esfuerzos académicos y sociales, esta conferencia confirmó la idea de que la ES es un bien público y un derecho humano, y que el estado debe garantizar el acceso universal permanente. Además, la ES responde a los ODS de forma integrada dada la complejidad social, económica, política, educativa, cultural, lingüística, biológica y geográfica de la región. Es inclusiva al considerar en los sistemas e Instituciones de ES género, edad, situación socioeconómica, orientación sexual, discapacidad, religión y situaciones de desplazamiento forzado. La CRES 2018 asumió la responsabilidad de las universidades para lograr y avanzar en estos objetivos como un tema fundamental, considerando los desafíos planteados por las escasas condiciones económicas y gran desigualdad (un tema central de los ODS), conflictos, violencia desenfrenada, aumento de la migración y la inequidad en los sistemas educativos.

Algunos principios para el abordaje integral de los ODS, en particular dos de sus estrategias vinculadas a la digitalización en las universidades son: 1) Fomentar la utilización de datos estadísticos con el fin de establecer diagnósticos y líneas de trabajo para la toma de decisiones y definición de políticas públicas de alcance nacional y regional; y 2) Extender la utilización de las TIC en propuestas innovadoras de la enseñanza en las IES para garantizar una formación de calidad con pertinencia e inclusión de diferentes sectores sociales.

Entendemos que estos ejes son parte clave de la transformación digital de las universidades, siendo necesario su abordaje desde una perspectiva crítica sobre el lugar que ocupa la tecnología en la ES y las tendencias que la interpelan, en particular en lo que respecta a la integración de la Inteligencia Artificial (IA), como eje central en la Cuarta Revolución Industrial, que impactará todas las áreas de la sociedad. La UNESCO respecto a los sistemas de $\mathrm{IA}^{6}$ destaca pueden ser de gran utilidad para la humanidad y que todos debieran gozar de sus beneficios, a la vez que suscitan preocupaciones éticas fundamentales, en relación con los sesgos que pueden llegar a provocar desigualdad, exclusión, amenazando la diversidad cultural y social, y la igualdad de género. Reconociendo al mismo tiempo las diferentes circunstancias de los distintos países, en particular los de América Latina y el Caribe, que se enfrentan a una aceleración del uso de las TICs y la IA, presentando importantes desafíos y oportunidades para las sociedades. Esta propuesta recoge las recomendaciones preliminares de la UNESCO en referencia a tener en cuenta que los riesgos y las preocupaciones éticas no deberían obstaculizar la innovación, sino estimular nuevas prácticas de investigación e innovación responsables, en las que el desarrollo y la utilización de la IA se fundamenten en valores morales y en la reflexión ética.

En este contexto de desigualdad social y muchas dificultades, para brindar una educación accesible e igualitaria para todos, y ante la posibilidad que brindan las investigaciones conjuntas

\footnotetext{
5 https://www.iesalc.unesco.org/2018/12/13/informe-general-de-la-cres-2018/

${ }^{6} \mathrm{https}: / / \mathrm{www}$.espacioiaunesco.org/
} 
entre los países de la región sobre el uso de las TICs en el contexto educativo, surgió la necesidad de crear una comunidad que conectando investigadores y educadores de distintos países de la región, pueda explorar un mayor uso de objetos de aprendizajes, así como también de distintas tecnologías incluyendo técnicas de IA, para mejorar el nivel educativo de la región.

Latinoamérica enfrenta la problemática de tener muy pocas iniciativas que ofrezcan, de forma global, apoyo a proyectos colaborativos de investigación y desarrollo de estrategias y políticas para promover la educación digital abierta y el uso de recursos educativos abiertos. Este artículo muestra un abordaje a esta problemática a través de la conformación de una red de investigadores latinoamericanos, mostrando los eventos y proyectos que se han logrado realizar en este marco, en el área de Informática en Educación.

En este sentido, es el objetivo de este artículo presentar a la comunidad LACLO y además, describir sintéticamente distintos proyectos y redes que han surgido a través de la sinergia de vínculos entre profesores e investigadores que trabajan en tecnologías educativas, muchos de ellos derivados de esa comunidad. Se pretende presentar así distintas miradas y especialidades, compartiendo problemáticas regionales con las particularidades propias de cada país, pero con el objetivo de poder contribuir a que una educación de calidad sea más accesible para todas y todos. A través del trabajo y desarrollo de esta comunidad, y las redes y proyectos vinculados con ella, se pretende mostrar el potencial de las sinergias que se pueden generar en estas redes de colaboración en nuestra región, que permiten sumar esfuerzos, compartir experiencias y tecnologías, con el fin de encontrar soluciones a problemas complejos y diversos que presentan nuestros países, pero que tienen muchos puntos en común.

\section{LACLO: una comunidad, una conferencia}

\subsection{LACLO: la comunidad}

En Latinoamérica siempre hubo la necesidad de compartir, unir esfuerzos y crecer juntos en muchas áreas, pero especialmente en aquellas que podrían conducirnos a mejorar el nivel de educación de nuestros pueblos. En este sentido, los pilares para una nueva era educativa se encuentran en etapa de desarrollo y los países latinoamericanos deben mantener un foro activo para la discusión de las alternativas tecnológicas existentes, que se puedan aplicar a nuestros contextos regionales.

Es así como, en el año 2006, un grupo de docentes investigadores de la Escuela Superior Politécnica del Litoral (ESPOL), organizó en Guayaquil (Ecuador) la $1^{a}$ Conferencia Latinoamericana de Objetos de Aprendizaje, a la cual llamaron LACLO 2006. Acudieron a la convocatoria tan solamente una decena de investigadores de pocos países de la región, la gran mayoría desconocidos entre ellos, pero conscientes de la necesidad de creación de un foro activo, de discusión continua de sus investigaciones y mejores prácticas sobre tecnologías educativas y del aprendizaje. Este foro permanente se convirtió en la Comunidad Latinoamericana de Objetos de Aprendizaje y Tecnologías de la Educación (LACLO), la cual continuó creciendo a lo largo de la región, contando ahora con miembros de otras regiones que activamente participan en las actividades desarrolladas.

Durante el bienio 2011-2012, la Comunidad fue incluida dentro del programa COMCLARA2011; proyecto diseñado por la RedCLARA ${ }^{7}$ (Red de Cooperación Latino Americana de Redes Avanzadas), para fortalecer los vínculos de trabajo en el ámbito de los intereses de investigación, consolidando las relaciones de los investigadores y empleando

\footnotetext{
${ }^{7}$ https://www.redclara.net/
} 
recursos de telecomunicaciones e informática que pueden usarse a través de Internet Avanzado, expandido por toda Latinoamérica, gracias a otros proyectos de RedCLARA bajo financiamiento europeo. Aunado a lo anterior, la Comunidad ha logrado alianzas con importantes organizaciones de la región, así como europeas, como Fundación Ariadne ${ }^{8}$ y GLOBE $^{9}$ (Global Learning Objects Brokered Exchange); desarrolló el repositorio LAFLOR (Repositorio Latinoamericano de Objetos de Aprendizaje). De igual forma, sus miembros han desarrollado varios proyectos financiados por entes regionales e internacionales y en especial, por la Comisión Europea, junto a investigadores de Europa. Algunos de esos proyectos van a ser detallados a continuación.

Hoy en día, LACLO es una Comunidad madura, conformada por cientos de investigadores de Latinoamérica, así como algunos de Norteamérica y Europa. Sus miembros continúan trabajando activamente y de forma colaborativa, desarrollando proyectos, intercambiando conocimiento y discusiones entorno a las tecnologías educativas.

\subsection{LACLO: la conferencia}

Como ya se ha expuesto anteriormente, el objetivo de la $1^{a}$ Conferencia Latinoamericana de Objetos de Aprendizaje fue la creación de un foro permanente, donde investigadores de Latinoamérica pudieran discutir sus investigaciones y mejores prácticas acerca de las tecnologías de aprendizaje, así como fomentar la cooperación entre los grupos de trabajo existentes, que en aquel momento todavía no se conocían. Quince años pasaron y LACLO ha logrado viajar con éxito a distintos países de Latinoamérica, a saber: Ecuador (Guayaquil, Loja), Chile (Valdivia, Santiago), Uruguay (Montevideo), Brasil (São Paulo, Maceió), México (Aguascalientes, Mérida, San José del Cabo), Colombia (Manizales), Costa Rica (San Carlos) y Argentina (La Plata). El infográfico de la Figura 4 muestra la cobertura geográfica y temporal de la conferencia.

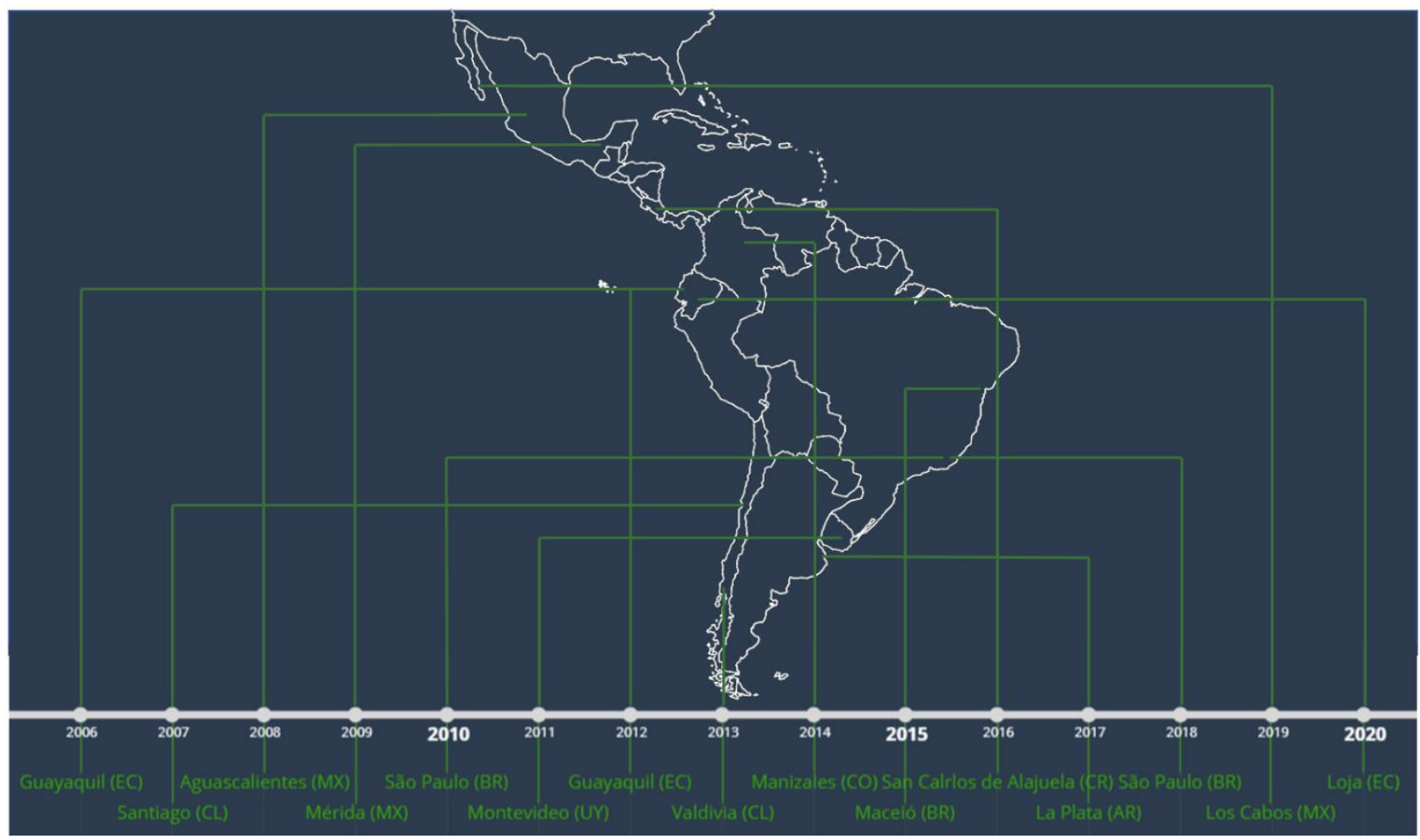

Figura 4. Alcance de la conferencia LACLO hasta el año 2020 (Fuente: autores)

\footnotetext{
${ }^{8}$ https://www.ariadne-eu.org/

9 https://wiki.p2pfoundation.net/Global_Learning_Objects_Brokering_Exchange
} 
La conferencia que inicialmente se restringía a los objetos de aprendizaje, poco a poco se convirtió en un foro más amplio para la discusión de todos los aspectos tecnológicos y pedagógicos relacionados a las tecnologías educativas. Un estudio sistemático realizado en 2017 sobre las áreas de investigación de los trabajos presentados en las ediciones de la conferencia en el período 2009-2016 (Deco et al. 2017), permitió observar la gran diversidad y actualidad de los diferentes temas presentados, entre los cuales destacan: sistemas de recomendación, repositorios educativos, analíticas de aprendizaje y minería de datos educativos, clases invertidas, juegos serios y realidad virtual y aumentada, aprendizaje personalizado, adaptabilidad y accesibilidad, cursos masivos en línea (MOOCs), experiencias docentes, entre otras. Un análisis breve de las palabras-clave de los artículos aceptados en la edición de 2020 revela que muchos de esos temas siguen siendo de interés para la comunidad, mientras que nuevos tópicos han aparecido, como se puede verificar en la nube de palabras de la Figura 5, en que temas como la realidad virtual y aumentada aparecen junto a gamificación, geolocalización, sensores y también investigaciones sobre los impactos de la pandemia de COVID-19 en la Educación.

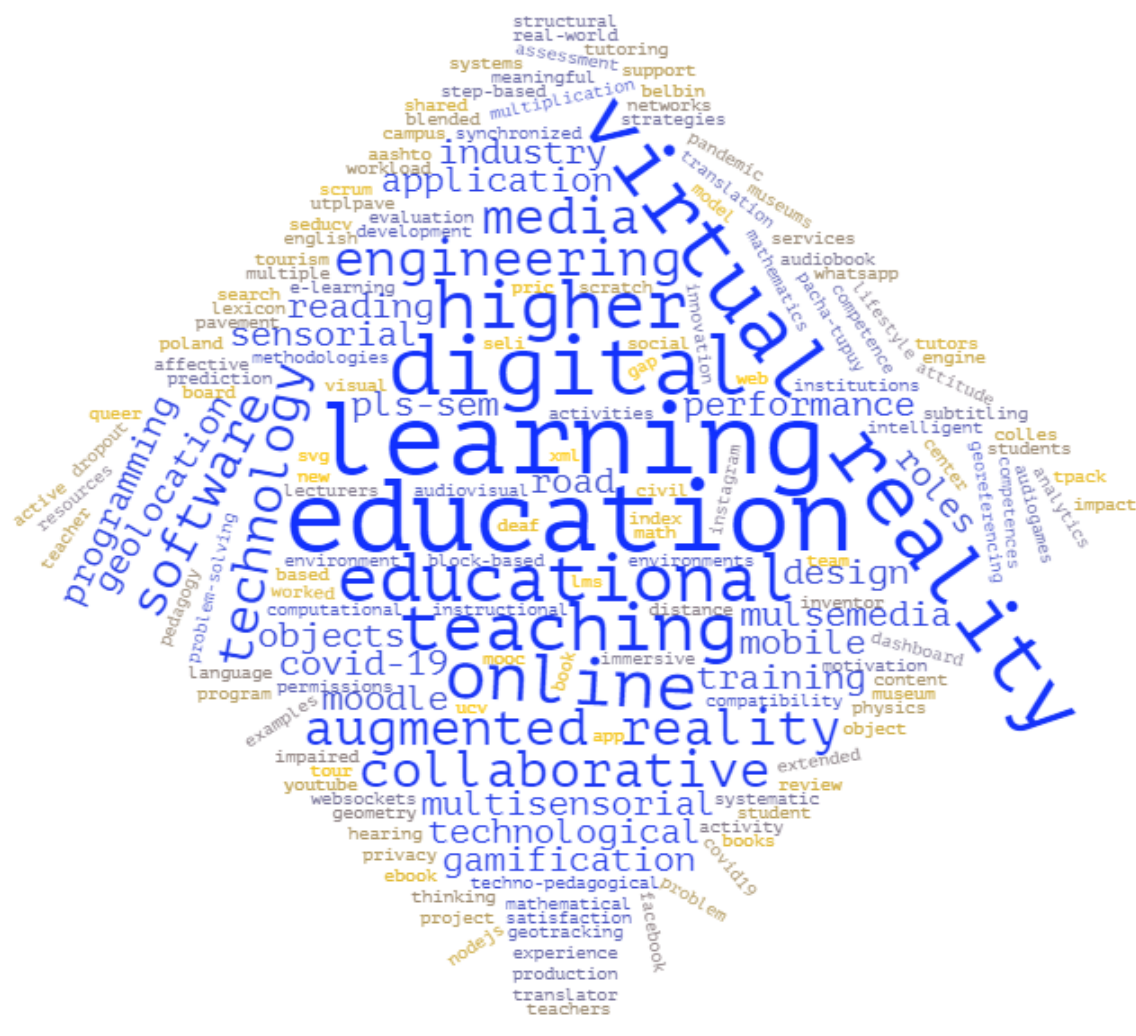

Figura 5. Nube con las palabras-clave de la conferencia LACLO 2020 (Fuente: autores)

Hay que destacar que, en diferentes ocasiones, la conferencia se agregó a otros eventos locales, entre los cuales se destacan la edición de 2015 que ha sido celebrada en conjunto con el CBIE (Congreso Brasileño de Informática Educativa), que es el evento científico más importante en el área de tecnologías educativas en el contexto brasileño. De la misma manera, la conferencia LACLO se unió dos veces a la conferencia CLEI (Centro Latinoamericano de Estudios en Informática), en 2018 y 2020 - experiencia que se pretende repetir en 2022 en la edición colombiana. También hay que destacar que por razón de la pandemia de COVID-19, la conferencia CLEI-LACLO de 2020 ha sido realizada por la primera vez de manera totalmente remota, como planea ser la edición peruana de 2021. 
La inclusión gradual de nuevos temas en la conferencia llevó a su redefinición como "Conferencia Latinoamericana de Tecnologías de Aprendizaje". A partir del año 2016, en su edición XI la conferencia ha publicado sus memorias en acceso de IEEEXplore ${ }^{10}$ con el fin de lograr mejorar los índices de impacto y el interés en la comunidad de investigación. Esta estrategia de publicación en IEEEXplore permitió apoyar a los investigadores que requieren por parte de sus universidades financiamientos en participación de eventos de impacto académico medible.

Enfocada en la integración de la comunidad latinoamericana, la conferencia se constituye hoy en una importante herramienta utilizada por la comunidad para fortalecer los lazos entre los distintos países, siempre agregando los diferentes grupos de investigación que gradualmente se suman a la comunidad. Por ejemplo, el estudio de la colaboración científica dentro de la propia comunidad realizado por Cechinel (2013) identificó un aumento significativo de las redes de colaboración dentro de la conferencia, así como también encontró dos países latinoamericanos que participaban de la conferencia pero que todavía no estaban totalmente integrados en las redes de colaboración: Costa Rica y Perú. Esas informaciones ayudaron a la comunidad a llevar a cabo la conferencia en 2016 en Costa Rica, y a planear una futura conferencia LACLO en Perú (a desarrollarse en 2021). Sin embargo, la colaboración en el caso de la autoría de recursos educativos se presenta en LACLO asociada a los ámbitos territoriales, articulados por las instituciones de las que forman parte los individuos que la componen (Rodés Paragarino et al., 2016) que se agrupan en torno a la creación colaborativa de recursos educativos. Esta diferencia no es menor, dados los fines vinculados al acceso y reutilización de recursos educativos, que se enfocan a trascender lo territorial. Esto ha conducido a una intensa red de colaboración regional a partir de numerosos proyectos orientados al desarrollo de redes de docentes e investigadores, así como al desarrollo profesional docente.

\section{Proyectos y redes de investigación}

A lo largo de los años, un número considerable de proyectos y redes de investigación multilateral y transnacional emergieron de los lazos construidos entre los miembros de la comunidad. La Tabla 1 presenta una visión general de algunas de las principales iniciativas y en la sección se describen sus objetivos, resultados e impactos. A las iniciativas, redes y proyectos que siguen activos, se incluye la URL donde se puede consultar más informaciones.

Tabla 1. Iniciativas que tuvieran apoyo o emergieron de la comunidad LACLO

\begin{tabular}{|c|c|c|c|}
\hline Iniciativa & Financiación & Instituciones (País) & Período \\
\hline $\begin{array}{l}\text { LATIn (Latin American } \\
\text { Open Textbook Initiative } \\
\text { - Iniciativa } \\
\text { Latinoamericana de } \\
\text { Libros de Texto Abiertos) }\end{array}$ & $\begin{array}{l}\text { Programa ALFA III } \\
\text { de la Unión Europea }\end{array}$ & $\begin{array}{l}\text { Univ. Nacional de Rosario (Argentina), Univ. } \\
\text { Presbiteriana Mackenzie (Brasil), Univ. Leuven } \\
\text { (Bélgica), Univ. Austral de Chile (Chile), Univ. } \\
\text { del Cauca (Colombia), Escuela Superior } \\
\text { Politécnica del Litoral (Ecuador), Univ. de } \\
\text { Alcalá de Henares(España), Univ. Paul } \\
\text { Sabatier (Francia), Univ. Autónoma de } \\
\text { Aguascalientes (México), Univ. Católica de } \\
\text { San Pablo (Perú), Univ. de la República } \\
\text { (Uruguay), y Univ. Central de Venezuela } \\
\text { (Venezuela) }\end{array}$ & $\begin{array}{l}2011- \\
2014\end{array}$ \\
\hline
\end{tabular}

${ }^{10} \mathrm{https} / / /$ ieeexplore.ieee.org/xpl/conhome/1816004/all-proceedings 


\begin{tabular}{|c|c|c|c|}
\hline $\begin{array}{l}\text { RIURE (Red } \\
\text { Iberoamericana para la } \\
\text { Usabilidad de Recursos } \\
\text { Educativos) }\end{array}$ & $\begin{array}{l}\text { CYTED } \\
(513 R T 0471)\end{array}$ & $\begin{array}{l}\text { Univ. de Vigo (España), Univ. de Santiago de } \\
\text { Compostela (España), Univ. Complutense de } \\
\text { Madrid (España), Instituto Superior de Eng. do } \\
\text { Porto (Portugal), Univ. Federal de Pelotas } \\
\text { (Brasil), Univ. Presbiteriana Mackenzie } \\
\text { (Brasil), Univ. Nacional de Rosario } \\
\text { (Argentina), Univ. de la República (Uruguay), } \\
\text { Escuela Superior Politécnica del Litoral } \\
\text { (Ecuador), Univ. Técnica Particular de Loja } \\
\text { (Ecuador) }\end{array}$ & $\begin{array}{l}2013- \\
2016\end{array}$ \\
\hline $\begin{array}{l}\text { ESVI-AL (Educación } \\
\text { Superior Virtual } \\
\text { Inclusiva - América } \\
\text { Latina) }\end{array}$ & $\begin{array}{l}\text { Programa ALFA III } \\
\text { de la Unión Europea }\end{array}$ & $\begin{array}{l}\text { UG (Guatemala), Univ. de la República } \\
\text { (Uruguay), Univ. Tecnológica Particular de } \\
\text { Loja (Ecuador), UC (Perú), Univ. Católica del } \\
\text { Norte (Colombia), UP (El Salvador), Univ. } \\
\text { Nacional de Asunción (Paraguay), Univ. de } \\
\text { Alcalá de Henares (España), Univ. de Lisboa } \\
\text { (Portugal) y Univ. de Ciencias Aplicadas } \\
\text { Metropolitana de Helsinki (Finlandia) }\end{array}$ & $\begin{array}{l}2011- \\
2015\end{array}$ \\
\hline $\begin{array}{l}\text { REMAR (Red Mercosur } \\
\text { para la Accesibilidad y } \\
\text { la generación } \\
\text { colaborativa de } \\
\text { Recursos Educativos } \\
\text { Abiertos }^{11} \text { ) }\end{array}$ & $\begin{array}{l}\text { Programa del Sector } \\
\text { Educativor del } \\
\text { Mercosur (PASEM) }\end{array}$ & $\begin{array}{l}\text { Universidad Nacional de La Plata (Argentina), } \\
\text { Universidad de la República (Uruguay), } \\
\text { Universidad Federal de Pelotas (Brasil), } \\
\text { Universidad Nacional de Asunción (Paraguay) }\end{array}$ & $\begin{array}{l}2013- \\
2015\end{array}$ \\
\hline DIIA & $\begin{array}{lr}\text { ANII } & \text { (Agencia } \\
\text { Nacional } & \text { de } \\
\text { Investigación } & \mathrm{e} \\
\text { Innovación, } \\
\text { Uruguay) }\end{array}$ & $\begin{array}{l}\text { Universidad de la República (Uruguay), } \\
\text { Universidad de las Américas, Puebla (México). }\end{array}$ & $\begin{array}{l}2017- \\
2018\end{array}$ \\
\hline Modelos Predictivos & $\begin{array}{lr}\text { ANII } & \text { (Agencia } \\
\text { Nacional } & \text { de } \\
\text { Investigación } & \text { e } \\
\text { Innovación, } \\
\text { Uruguay) }\end{array}$ & $\begin{array}{l}\text { Universidad de la República (Uruguay), } \\
\text { Escuela Superior Politécnica del Litoral } \\
\text { (Ecuador), Universidad Federal de Santa } \\
\text { Catarina (Brasil) }\end{array}$ & $\begin{array}{l}2016- \\
2018\end{array}$ \\
\hline $\begin{array}{l}\text { SELI (Smart Ecosystem } \\
\text { for Learning and } \\
\text { Inclusion }^{12} \text { ) }\end{array}$ & ERANET-LAC & $\begin{array}{l}\text { Univ. of Eastern Finland (Finlandia), Univ. } \\
\text { Presbiteriana Mackenzie (Brasil), Univ. de la } \\
\text { República (Uruguay), Univ. del Azuay } \\
\text { (Ecuador), Univ. Central "Marta Abreu" de Las } \\
\text { Villas (Cuba), Univ. Federico Henríquez y } \\
\text { Carvajal (República Dominicana), Univ. } \\
\text { Mayor de San Simón (Bolivia), } \\
\text { Univ. Tecnológica de Panamá, (Panamá), } \\
\text { Pedagogical University of Cracow, Faculty of } \\
\text { Education (Polonia), Hacettepe University } \\
\text { (Turquía) }\end{array}$ & $\begin{array}{l}2018- \\
2021\end{array}$ \\
\hline
\end{tabular}

\footnotetext{
${ }^{11} \mathrm{https}$ ://proyectoremar.wordpress.com/
}

12 https://seliproject.org 


\begin{tabular}{|c|c|c|c|}
\hline $\begin{array}{l}\text { HCI-Collab }{ }^{13} y \\
\text { VG-Collab } \\
\text { 14 }\end{array}$ & $\begin{array}{l}\text { AUIP (Asociación } \\
\text { Universitaria } \\
\text { Iberoamericana de } \\
\text { Posgrados) }\end{array}$ & $\begin{array}{l}\text { Univ. Nacional de La Plata, Univ. Nacional de } \\
\text { la Matanza, Univ. Nacional de San Juan, Univ. } \\
\text { Kennedy(Argentina), Univ. Presbiteriana } \\
\text { Mackenzie, Univ. Federal do Rio Grande do } \\
\text { Sul (Brasil); Pontificia Univ. Católica de } \\
\text { Valparaíso, Univ. de Playa Ancha, Universidad } \\
\text { de la Frontera (Chile); Univ. del Cauca, Univ. } \\
\text { San Buenaventura, Univ. Cooperativa de } \\
\text { Colombia) (Colombia); Univ. Nacional de } \\
\text { Costa Rica, Tecnológico de Costa Rica (Costa } \\
\text { Rica), Univ. de Cs. Informáticas (Cuba); Univ. } \\
\text { Técnica Particular de Loja (Ecuador); Univ. de } \\
\text { Lleida, Univ. Zaragoza, Univ. de la Laguna, } \\
\text { Univ. de Granada (España); Univ. Autónoma } \\
\text { de Aguascalientes, Univ. Autónoma de Puebla } \\
\text { (México); Univ. Tecnológica de Panamá; Univ. } \\
\text { Portucalense (Portugal); Univ. Central de } \\
\text { Venezuela }\end{array}$ & $\begin{array}{l}2019 \text { - } \\
\text { en curso }\end{array}$ \\
\hline $\mathbf{L A L A}^{15}$ & ERASMUS+ & $\begin{array}{l}\text { Miembros Fundadores: Pontificia Univ. } \\
\text { Católica de Chile (Chile), Escuela Superior } \\
\text { Politécnica del Litoral (Ecuador), Univ. de } \\
\text { Cuenca (Ecuador), Univ. Carlos III (España), } \\
\text { Univ. de Edimburgo (Escocia), KU Leuven } \\
\text { (Bélgica) }\end{array}$ & $\begin{array}{l}2018- \\
\text { en curso }\end{array}$ \\
\hline
\end{tabular}

A continuación, se detallan cada uno de esos proyectos y redes.

\subsection{LATIn}

El Proyecto LATIn (Latin American Open Textbook Initiative - Iniciativa Latinoamericana de Libros de Texto Abiertos) (Ochoa, Silveira \& Sprock, 2011) fue desarrollado entre los años 2011 y 2014, en el marco del proyecto ALFA III financiado por la Unión Europea. Participaron investigadores de la Comunidad de nueve países de América Latina, con el apoyo de tres universidades europeas, de España, Francia y Bélgica.

Este proyecto abordó el problema del alto costo de los libros de texto para la Educación Superior, inicialmente en América Latina, pero siguiendo pautas que consideraron variables como: país, idioma, cultura y región. Una de las acciones principales del proyecto LATIN es la creación y diseminación de una iniciativa para la producción colaborativa de Libros de Textos Abiertos para la Educación Superior. Esta iniciativa ha permitido apoyar a los profesores de diferentes Instituciones de Educación Superior de Latinoamérica para que de forma colaborativa con colegas de otras instituciones de la región puedan crear capítulos y libros de texto diseñados para cubrir las necesidades de sus cursos. Se planteó una visión amplia de los libros digitales, de modo que estos libros estén disponibles para que los estudiantes los lean, impriman y compartan, y para que otros profesores los adapten, traduzcan, distribuyan y mezclen nuevamente - siguiendo así, los principios de REA (Recursos Educativos Abiertos). El proyecto tomó en cuenta los temas pedagógicos, tecnológicos y académicos necesarios para que dicha iniciativa funcione exitosamente en los países de Latinoamérica.

\footnotetext{
${ }^{13}$ https://hci-collab.com

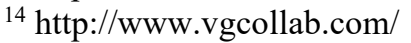

${ }^{15} \mathrm{https}: / /$ www.lalaproject.org/
} 
El Proyecto se planificó en torno a tres ejes principales (Deco et al., 2013) (Silveira et al., 2013a) guiados por las cuestiones pedagógicas, tecnológicas y políticas, que deberían ser consideradas para que este tipo de iniciativa tuviera éxito en el contexto de Latinoamérica. Estos ejes están estrechamente relacionados, estableciendo un ecosistema digital (Silveira et al, 2013b). El primer eje, incluyó los aspectos teóricos y metodológicos, donde se diseñó una metodología para la creación colaborativa de libros de texto abiertos, con énfasis en aspectos legales y procesos colaborativos (Casali et al., 2012). El segundo eje, tecnológico, incluyó el diseño e implementación de las plataformas tecnológicas para apoyar la creación colaborativa, adaptación, combinación y reutilización de libros abiertos (Ochoa et al., 2013). El tercer y último eje, consideró las estrategias para la implementación y adopción de esta iniciativa en las instituciones de educación superior de la región (Rodés et al., 2013).

Las plataformas computacionales, incluidas en el segundo eje, incluyeron una plataforma social para la formación de grupos de escritura y una plataforma de escritura colaborativa, ambas implementadas bajo la filosofía de mashups y puestas a disposición mediante licencias abiertas. Estas plataformas permitieron el surgimiento de 94 comunidades de escritores, las cuales propusieron 47 libros de texto abiertos. De estas propuestas, se seleccionaron 25 para ser desarrolladas en colaboración por 144 profesores-autores. Posteriormente, una vez elaborados estos libros colaborativos, fueron utilizados y evaluados por 186 profesores y 1.835 alumnos.

La Tabla 2 sintetiza los principales logros de la red, así como los desafíos de investigación que todavía siguen, relacionados al tema principal de LATIn.

Tabla 2. Objetivos alcanzados y desafíos de investigación latentes sobre la temática de LATIn

\begin{tabular}{|c|c|c|}
\hline & Objetivos alcanzados & Desafíos de investigación latentes \\
\hline $\begin{array}{c}\text { LATIn } \\
(2011-2014)\end{array}$ & $\begin{array}{l}\text { - LATIn estimuló por primera vez la } \\
\text { discusión en el ámbito latinoamericano, } \\
\text { sobre la validez y calidad de los libros- } \\
\text { texto abiertos, conformando una } \\
\text { importante iniciativa en el marco de REA } \\
\text { en Latinoamérica. } \\
\text { - Fueron colectados datos en nueve países de } \\
\text { Latinoamérica sobre la necesidad y } \\
\text { aceptación de libros-texto abiertos, entre } \\
\text { profesores y estudiantes. } \\
\text { Fue definido un conjunto de metodologías } \\
\text { de soporte a la escritura colaborativa en el } \\
\text { ámbito académico. } \\
\text { Por medio de software libre, se creó un } \\
\text { mashup de herramientas de software para: } \\
\text { la conformación de redes de escritura, la } \\
\text { escritura colaborativa, la organización y } \\
\text { publicación de libros y su versionamiento. }\end{array}$ & $\begin{array}{l}\text { - Las estrategias de adopción de libros } \\
\text { abiertos colaborativos tienen que ser } \\
\text { negociadas individualmente con las } \\
\text { editoriales académicas, profesores y } \\
\text { cuerpo directivo de las instituciones. En } \\
\text { ese sentido, se hacen necesarios estudios } \\
\text { más profundos sobre organizaciones } \\
\text { académicas y sus dinámicas. } \\
\text { Actualmente, los mecanismos de } \\
\text { colaboración ya son más conocidos y } \\
\text { utilizados por profesores-autores. Las } \\
\text { metodologías de escritura colaborativa } \\
\text { propuestas por LATIn deberían ser } \\
\text { actualizadas, considerando el nuevo } \\
\text { escenario. La misma actualización debería } \\
\text { realizarse con el mashup de software } \\
\text { legado. } \\
\text { Las estrategias y mecanismos de } \\
\text { versionamiento de REA siguen siendo un } \\
\text { tópico importante de investigación. }\end{array}$ \\
\hline
\end{tabular}

El proyecto dejó importantes legados para toda Latinoamérica, en especial por ser una de las primeras iniciativas transnacionales en la región en dirección a la adopción de libros-texto como REA (Recursos Educativos Abiertos) (Silveira, 2016), pero en la actualidad se encuentra desactivado. Una asociación sin fines de lucro (Asociación LATIn) con sede en Montevideo, llegó a ser creada para dar soporte a la iniciativa. Algunos de los libros desarrollados en este proyecto aún pueden verse en https://rephip.unr.edu.ar/handle/2133/17682. 


\subsection{RIURE}

La Red RIURE (Red Iberoamericana para la Usabilidad de Recursos Educativos) es una Red Temática CYTED ${ }^{16}$ (513RT0471) que surgió con el propósito de mejorar la usabilidad de los Repositorios Educativos, permitiendo búsquedas más intuitivas y sencillas. Participaron de esta red siete grupos de investigación de Argentina, Brasil, Uruguay, Ecuador, Portugal, fue coordinada por la Universidad de Vigo, España, y se desarrolló en el período 2013-2016.

Los repositorios de objetos de aprendizaje (LORs - Learning Objects Repositories) evolucionaron desde la necesidad de compartir material educativo entre una comunidad de docentes y estudiantes. A pesar de que parece muy beneficioso para el proceso educativo, compartir y reusar material educativo a través de LORs no es una práctica todavía adoptada masivamente. Una de las principales razones es la gran cantidad de esfuerzo que requiere la indexación, búsqueda y reutilización final de estos recursos. Los repositorios actuales para almacenamiento y recuperación de objetos de aprendizaje poseen diferentes problemas de usabilidad que todavía necesitan de mejoras para aumentar la utilización de dichas herramientas y su consolidación como plataformas de búsqueda de estos recursos.

RIURE se desarrolló abordando los siguientes objetivos específicos: (1) Introducir aspectos pedagógicos en los repositorios para que sean tenidos en cuenta tanto en la catalogación como en la búsqueda; (2) Incorporar contenidos multimedia en los repositorios (por ej. vídeos, juegos y simulaciones); (3) Desarrollar un conjunto de métricas de relevancia y calidad, basadas en la información intrínseca y extrínseca de los recursos; (4) Introducir sistemas de recomendación basados en el perfil de usuario, material y contexto, que sean integrable a los principales entornos de aprendizaje; (5) Utilizar estructuras semánticas (tanto RDF como Linked Data) para representar los materiales, usuarios y contexto, de tal manera que se puedan interrelacionar y permitir utilizar nuevos algoritmos de búsqueda y razonamiento; y (6) Permitir que el repositorio educacional se enlace con otros repositorios de información semánticamente anotada, ampliando la cantidad de recursos de información a los que tendrían acceso los estudiantes y profesores.

La Tabla 3 presenta una síntesis sobre los principales logros de RIURE y los desafíos de investigación que todavía siguen vigentes.

Tabla 3. Objetivos alcanzados y desafíos de investigación latentes sobre la temática de RIURE

\begin{tabular}{|c|c|c|}
\hline & Objetivos alcanzados & Desafíos de investigación latentes \\
\hline $\begin{array}{c}\text { RIURE } \\
(2013-2016)\end{array}$ & $\begin{array}{l}\text { - RIURE planteó una importante } \\
\text { iniciativa respecto a la usabilidad } \\
\text { de los repositorios de recursos } \\
\text { educativos en ámbito } \\
\text { iberoamericano. } \\
\text { - Guías de buenas prácticas para la } \\
\text { creación, mantenimiento y } \\
\text { evolución de repositorios fueron } \\
\text { producidos y puestos a testeo en } \\
\text { distintas situaciones. } \\
\text { RIURE estableció un modelo } \\
\text { automático de determinación de } \\
\text { calidad de recursos educativos } \\
\text { basándose en estrategias de } \\
\text { Minería de Datos. }\end{array}$ & $\begin{array}{l}\text { - El contexto de los repositorios de materiales } \\
\text { educativos sufrió distintos cambios desde entonces, } \\
\text { con nuevas formas de publicación no indexada de } \\
\text { contenido, nuevos modelos de licencias y distintos } \\
\text { formatos tecnológicos, con soporte más estable a } \\
\text { distintos contenidos multimedia. } \\
\text { - Aunque los sistemas de recomendación han logrado } \\
\text { muchos avances en los últimos cinco años, sigue } \\
\text { siendo un importante tema de investigación, ya que } \\
\text { se torna necesario desarrollar mecanismos que } \\
\text { puedan proteger el usuario, recomendando sin } \\
\text { violar su privacidad. Avances en el área de } \\
\text { Ciencias de datos están cambiando ese escenario. } \\
\text { La búsqueda y recuperación, incluyendo la } \\
\text { búsqueda semántica, de contenido educativo de } \\
\text { calidad, permanece como un desafío de } \\
\text { investigación. }\end{array}$ \\
\hline
\end{tabular}

${ }^{16} \mathrm{http}: / /$ www.cyted.org/ 
Algunos de los avances alcanzados pueden verse en distintas publicaciones del grupo (Casali et al. 2013; Casali et al., 2015; Rodes et al., 2015; Cechinel et al., 2016; Lemos et al., 2016). En el desarrollo del proyecto, se buscó sinergia con la comunidad LACLO y con el Proyecto LATIn, ya que esos dos ocurrieron parcialmente al mismo tiempo.

\subsection{ESVI-AL}

El proyecto ESVI-AL (Educación Superior Virtual Inclusiva - América Latina) fue un proyecto financiado por el programa ALFA III de la Unión Europea, ejecutado entre los años 2011 y 2015. En este proyecto participaron diez universidades, entre sus participantes se han destacado colaboraciones vinculadas a LACLO.

El Proyecto ESVI-AL se orientó a mejorar la accesibilidad de la educación superior virtual, a través de la creación e implantación de metodologías que establezcan un modelo de trabajo para el cumplimiento de requisitos y estándares de accesibilidad en el contexto de la formación virtual, especialmente a través de la Web. Entre sus principales contribuciones se encuentra la creación de la Red de Cooperación y Observatorio de Accesibilidad en la Educación y Sociedad Virtual ESVI-AL, que continúa otorgando sostenibilidad a las acciones desarrolladas, entre las que se destaca la creación de una Guía Metodológica y un Modelo de Acreditación de la Educación Virtual Accesible, la realización de cursos accesibles dirigidos a diferentes públicos y la realización de congresos y conferencias como el Congreso Internacional sobre Aplicación de Tecnologías de la Información y Comunicaciones Avanzadas (ATICA).

La Guía Metodológica ${ }^{17}$ ha sido concebida como un instrumento de apoyo para todos los involucrados en proyectos educativos virtuales accesibles, principalmente para los docentes, pero también para el personal de gestión, administración y apoyo técnico de las instituciones que pretendan implantar actividades formativas virtuales inclusivas, en las que puedan participar en igualdad de condiciones estudiantes sin o con discapacidad. En su segunda edición se presenta una propuesta de los procesos que deberían implantarse en una institución de educación superior, pero también en cualquier organización o empresa de formación virtual, que esté comprometida con una educación inclusiva de calidad. La segunda edición se ha realizado a partir de la experiencia de impartir e implementar los procesos propuestos en diversos talleres en varios países en América Latina. Esta nueva versión busca orientar al lector en los productos que debe obtener para cada una de las tareas y relaciona claramente qué técnicas y métodos facilitan su obtención, y muy importante, que métricas permiten verificar su calidad en base a criterios de referencia establecidos.

En conjunto con la Guía ESVI-AL se presenta un modelo de Acreditación ESVI-AL, dicho modelo propuesto facilita la elaboración de auditorías que permitan el diagnóstico de cumplimiento de normas de accesibilidad y la mejora de la capacidad de madurez, respecto a la accesibilidad, de las instituciones de educación superior y en general para organizaciones de educación.

A continuación, la Tabla 4 presenta una síntesis sobre los principales logros de ESVI-AL y los desafíos de investigación que todavía siguen vigentes.

${ }^{17}$ La guía se puede descargar de: http://www.esvial.org/guia/wp-content/uploads/2015/03/2015_GUIAESVIAL_2da_Edicion.pdf 
Tabla 4. Objetivos alcanzados y desafíos de investigación latentes sobre la temática de ESVI-AL

\begin{tabular}{|c|c|c|}
\hline & Objetivos alcanzados & Desafíos de investigación latentes \\
\hline $\begin{array}{c}\text { ESVI-AL } \\
(2011-2015)\end{array}$ & $\begin{array}{l}\text { - ESVI-AL permitió la definición de una } \\
\text { Guía Metodológica con referentes de } \\
\text { accesibilidad de la educación superior } \\
\text { virtual. } \\
\text { - Se estableció la Red de Cooperación y } \\
\text { Observatorio de Accesibilidad en la } \\
\text { Educación y Sociedad Virtual ESVI-AL. } \\
\text { Fue propuesto el modelo de Acreditación } \\
\text { ESVI-AL, que facilitaría la conducción } \\
\text { de auditorías para el diagnóstico de } \\
\text { cumplimiento de normas de accesibilidad } \\
\text { en el ámbito educativo. }\end{array}$ & $\begin{array}{l}\text { - Los temas de accesibilidad en entornos } \\
\text { virtuales siguen siendo importantes en el } \\
\text { contexto de investigación. } \\
\text { Aunque el W3C (World Wide Web } \\
\text { Consortium) haya establecido las WCAG } \\
\text { (Web Content Accessibility Guidelines), } \\
\text { hay un gran desafío en lo que se refiere a } \\
\text { la adopción efectiva de aspectos de } \\
\text { accesibilidad como requerimientos } \\
\text { fundamentales en el diseño de soluciones } \\
\text { educativas y no solamente como features } \\
\text { opcionales. }\end{array}$ \\
\hline
\end{tabular}

En ESVI-AL, se buscó la creación de planes de estudio para las necesidades sociales, de sostenibilidad y empleabilidad de las personas con discapacidades físicas de los países de Latinoamérica. En la ejecución del proyecto, se estableció sinergia con la comunidad LACLO y el Proyecto LATIn. Muchos de los aspectos trabajados en ESVI-AL están hoy día cubiertos por la iniciativa HCI-Collab, que se presenta más adelante.

\subsection{REMAR}

La red REMAR (Red Mercosur para la Accesibilidad y la generación colaborativa de Recursos Educativos Abiertos $^{18}$ ) (Díaz et al., 2014), fue una iniciativa de docentes de la comunidad LACLO, coordinados por la Profa. Alicia Díaz de la Universidad Nacional de La Plata, con participación de la Universidad de la República, Uruguay, la Universidad Federal de Pelotas, Brasil y la Universidad Nacional de Asunción, Paraguay, contando con financiamiento del Programa del Sector Educativo del Mercosur (PASEM), en el período 2013-2015.

REMAR ofreció una serie de 4 (cuatro) talleres de trabajo conjunto como un espacio virtual de comunicación donde los docentes de la región interesados en el tema de formación inclusiva pudieron compartir experiencias y disponer de herramientas que facilitaban el uso y el desarrollo de contenidos educativos abiertos y accesibles. Los 4 talleres alcanzaron más de 200 docentes de distintos países de Latinoamérica (Argentina, Brasil, El Salvador, Paraguay y Uruguay) y abordaron los siguientes tópicos: Recursos Educativos Abiertos Accesibles (REAAs), Creación Colaborativa de Contenidos (herramientas, práctica de creación de recursos), Accesibilidad, y Descripción de Metadatos (IEEE LOM).

Tabla 5. Objetivos alcanzados y desafíos de investigación latentes sobre la temática de REMAR

\begin{tabular}{|c|c|c|}
\hline & Objetivos alcanzados & Desafíos de investigación latentes \\
\hline $\begin{array}{c}\text { REMAR } \\
(2013-2015)\end{array}$ & $\begin{array}{l}\text { - REMAR fue un proyecto de } \\
\text { sensibilización y capacitación de } \\
\text { docentes, proponiendo talleres sobre } \\
\text { distintos temas. } \\
\text { Mas allá de los talleres de capacitación, } \\
\text { REMAR conformó una red de apoyo a } \\
\text { docentes de cinco países preocupados } \\
\text { con prácticas educativas inclusivas. }\end{array}$ & $\begin{array}{l}\text { - Las temáticas principales de REMAR } \\
\text { siguen con brechas de investigación aún } \\
\text { por evolucionar, como por ejemplo los } \\
\text { aspectos de accesibilidad, colaboración y } \\
\text { recuperación de contenidos. } \\
\text { - Uno de los grandes desafíos prácticos se } \\
\text { encuentra en el proceso de capacitación } \\
\text { docente, más allá del simple uso de las } \\
\text { tecnologías disponibles, pero igual en lo } \\
\text { que se refiere a la sensibilización sobre } \\
\text { temas importantes en la práctica docente }\end{array}$ \\
\hline
\end{tabular}

\footnotetext{
18 https://proyectoremar.wordpress.com/
} 
La propuesta de REMAR estableció fuerte diálogo con las iniciativas ESVI-AL y LATIn, además de la comunidad LACLO. Se ha incentivado en las instituciones miembros y en otros participantes de los talleres, el deseo de seguir creando REAAs en los entornos de trabajo de los docentes, de conseguir en sus instituciones la implantación de los repositorios locales y de seguir manteniendo los lazos entre los docentes de áreas afines (Cechinel et al., 2015).

\subsection{Proyecto DIIA}

El proyecto DIIA: Descubrimiento de Interacciones que Impactan en el Aprendizaje, se desarrolló entre miembros de la comunidad LACLO que provenían de la Universidad de la República, Uruguay y la Universidad de las Américas, Puebla, México, en el período 2017-2018 con financiación de la Agencia ANII-Uruguay. El proyecto trabajó sobre la extracción de información relevante a partir de las distintas interacciones asociadas con un curso, tanto en los ambientes formales (Learning Management Systems) como en ambientes informales (redes sociales, Facebook). Para poder detectar patrones a partir de las interacciones generadas en las redes sociales, se propuso el uso de una representación basada en grafos donde se modelan los distintos tipos de interacciones posibles entre los actores de un curso (Cervantes et. al. 2018, Motz et al. 2018). Además de estudiar los patrones topológicos presentes en las distintas interacciones entre los principales actores de un curso, otra importante herramienta para entender el comportamiento social de estudiantes y docentes es el análisis de sentimientos basado en el contenido de los textos intercambiados, donde la idea es la de encontrar la polaridad del sentimiento (positivo, negativo o neutro) de las publicaciones o comentarios compartidos en los cursos para determinar el estado de ánimo de los distintos actores.

Para la plataforma DIIA se desarrolló un clasificador de textos supervisado que por medio de la extracción de características léxico-sintácticas como los trigramas de palabras con alta frecuencia de aparición, pueda diferenciar un sentimiento de otro. Este clasificador se basa en el entrenamiento de un modelo que busca las características presentes en un conjunto de datos de entrenamiento, para posteriormente buscar la existencia de los patrones en nuevos contenidos asociados a un curso.

Trabajos previos sobre análisis de sentimientos en los mensajes de los estudiantes (Buenaño-Fernandez et al. 2018) se limitan al análisis de los mensajes sin visualizar el impacto que tienen dentro del grupo tal como es planteado por las medidas en el grafo que provee DIIA. En este sentido, la propuesta del análisis de los patrones de las interacciones sociales de DIIA ofrece gran potencial para convertirse en una herramienta de análisis del aprendizaje colaborativo. Implementaciones de DIIA permitirían comparar el rendimiento académico de un estudiante en relación con las interacciones ocurridas en el grupo de aprendizaje en que se encuentra. La tabla 6 brinda una síntesis de los objetivos alcanzados y los desafíos latentes de investigación.

Tabla 6. Objetivos alcanzados y desafíos de investigación latentes sobre la temática de DIIA

\begin{tabular}{|c|c|c|}
\hline & Objetivos alcanzados & Desafíos de investigación latentes \\
\hline $\begin{array}{c}\text { DIIA } \\
(2017-2018)\end{array}$ & $\begin{array}{l}\text { - DIIA propuso un ambiente de software } \\
\text { para descubrir patrones semánticos de } \\
\text { interacción, tras la aplicación de analítica } \\
\text { del aprendizaje basada en los entornos } \\
\text { sociales provistos por dos plataformas. }\end{array}$ & $\begin{array}{l}\text { - El tema de analítica de aprendizaje para } \\
\text { descubrir patrones semánticos es un tema } \\
\text { importante de investigación. Uno de los } \\
\text { desafíos de su investigación en } \\
\text { Latinoamérica es la baja disponibilidad de } \\
\text { datos educativos y su falta de } \\
\text { estandarización. }\end{array}$ \\
\hline
\end{tabular}


Un primer prototipo de la plataforma DIIA ya está disponible desarrollada en software libre y puede ser usada a partir de la carga de datos de los espacios virtuales de aprendizaje ${ }^{19}$.

\subsection{Proyecto Modelos Predictivos}

El proyecto Modelos Predictivos ${ }^{20}$ (2016-2018) buscó la creación de un sistema de alerta temprana para riesgo académico en los estudiantes de educación secundaria pública de Uruguay. El proyecto fue integrado por miembros de la comunidad LACLO asociados a la Escuela Superior Politécnica del Litoral (ESPOL) de Ecuador, la Universidad Federal de Santa Catarina (UFSC), la Universidad de la República (UDELAR), y la Administración Nacional de Educación Pública de Uruguay (ANEP) y fue financiado por el Fondo Sectorial "Inclusión Digital: Educación con Nuevos Horizontes" - 2016 de la Agencia Nacional de Investigación e Innovación (ANII) de Uruguay.

Haciendo uso de técnicas de minería de datos educativos, el proyecto encontró un conjunto de patrones que ayudaron en el desarrollo de un prototipo de dashboard para el seguimiento de la trayectoria académica de los estudiantes y la identificación de los estudiantes en riesgo (Macarini et al., 2020). Junto al producto desarrollado, el proyecto también aportó con un conjunto de desafíos enfrentados hacia la implementación de iniciativas de analíticas de aprendizaje a nivel nacional (Macarini et al., 2019). La Tabla 7 presenta una síntesis de esto proyecto.

Tabla 7. Objetivos alcanzados y desafíos de investigación latentes sobre la temática de Modelos Predictivos

\begin{tabular}{|c|c|c|}
\hline & Objetivos alcanzados & Desafíos de investigación latentes \\
\hline $\begin{array}{c}\text { Modelos } \\
\text { Predictivos } \\
(2016-2018)\end{array}$ & $\begin{array}{l}\text { - El proyecto entregó el desarrollo de un } \\
\text { software que brindaba un dashboard de } \\
\text { seguimiento de trayectoria académica por } \\
\text { medio del uso de técnicas de minería de } \\
\text { datos. }\end{array}$ & $\begin{array}{l}\text { Muchos desafíos logísticos } \\
\text { enfrentados durante el desarrollo del } \\
\text { proyecto siguen siendo las principales } \\
\text { barreras para la implementación de } \\
\text { proyectos de Ciencia de Datos } \\
\text { Educativos en Latinoamérica, a saber: } \\
\text { la burocracia para obtener la } \\
\text { autorización para acceder a los datos; } \\
\text { la necesidad de mantener los datos } \\
\text { protegidos según las leyes de } \\
\text { protección de datos de cada país, lo } \\
\text { que añade una (necesaria) capa de } \\
\text { complejidad en la anonimización; la } \\
\text { falta de patrón y la inconsistencia en } \\
\text { las bases de datos; y la dificultad para } \\
\text { definir una metodología única para } \\
\text { exploración y análisis de los datos. }\end{array}$ \\
\hline
\end{tabular}

Actualmente, una extensión del proyecto sigue en desarrollo ampliando el conjunto de datos para la enseñanza primaria y secundaria técnica. En esta nueva etapa el proyecto cuenta con financiamiento del Banco Interamericano de Desarrollo (BID) (Contrato RG-T3450-P004) bajo la iniciativa fAIrLAC ${ }^{21}$ y con la participación de ANEP, UDELAR, UFSC y la Universidad de Nueva York (NYU).

\footnotetext{
19 Se puede acceder a una demo interactiva en http://www.diia.edu.uy con email guillermina@gmail.com y contraseña prototipo_para explorar las visualizaciones que se ofrecen.

$20 \mathrm{https} / /$ proeva.udelar.edu.uy/modelos-de-prediccion-para-la-determinacion-de-riesgo-academico/

${ }^{21}$ https://fairlac.iadb.org/
} 


\subsection{SELI}

SELI (Smart Ecosystem for Learning and Inclusion) (Martins et al., 2019) surge como un proyecto conjunto entre Latinoamérica y Europa, financiado bajo el marco de la convocatoria ERANET-LAC, desarrollado entre noviembre 2018 y diciembre 2021. Participan del proyecto universidades de Brasil, Cuba, Ecuador, Panamá, República Dominicana y Uruguay en Latinoamérica (y de Finlandia, Polonia y Turquía en Europa). Parte de los integrantes de Latinoamérica fueron originarios de la comunidad LACLO y lograron integrar los demás participantes desde la comunidad y la conferencia.

El proyecto desarrolla el concepto de los ecosistemas digitales inteligentes para el apoyo a la inclusión en el contexto educativo. El ecosistema se basa en tecnologías y arquitecturas recientes como blockchain y microsites, con estrategias metodológicas centradas en el estudiante, como el uso de digital storytelling, entre otras. Algunos de los resultados del proyecto han sido la construcción de una red blockchain educativa con nodos en Ecuador, Uruguay y Finlandia, implementación de asistentes para el diseño instruccional de cursos accesibles, así como varias tesis de posgrado desarrolladas en el marco del proyecto.

Por ser un proyecto aún en desarrollo, la Tabla 8 presenta los principales desafíos de investigación que lo mueven.

Tabla 8. Principales objetivos de investigación de SELI

\begin{tabular}{|c|l|}
\hline \multirow{2}{*}{ SELI } & Objetivos de investigación \\
\cline { 2 - 3 }$(2018-2021)$ & $\begin{array}{l}\text { SELI busca brindar acceso a la educación a personas con discapacidades y otros grupos } \\
\text { en vulnerabilidad, como ancianos o migrantes. En ese sentido, se apoya en estrategias de } \\
\text { desarrollo basado en Diseño Universal y técnicas descentralizadas, como el uso de } \\
\text { blockchain, en la conformación de un ecosistema digital para la educación e inclusión. } \\
\text { Igualmente, se busca aumentar la competencia de los docentes en el uso de las } \\
\text { tecnologías y mejorar la participación de los estudiantes a través de métodos innovadores, } \\
\text { por medio de talleres de sensibilización y capacitación en Europa y Latinoamérica. }\end{array}$ \\
\hline
\end{tabular}

Se obtuvieron, además, gracias al proyecto SELI, nuevos estudios cualitativos sobre la percepción que tienen los docentes y los estudiantes sobre el uso de las tecnologías en educación realizados de forma uniforme entre los países de Latinoamérica involucrados en el proyecto, como se observa en Tomczyk et al. $(2019,2020)$.

\subsection{HCI-Collab y VG-Collab}

La red HCI-Collab (Red Colaborativa para soportar los procesos de enseñanza-aprendizaje en el área de Interacción Humano-Computador a nivel Iberoamericano), surge como una iniciativa auspiciada por la AUIP (Asociación Universitaria Iberoamericana de Posgrados), la cual busca fomentar el área de HCI en Países Iberoamericanos. Esta red actualmente está conformada por 34 Universidades de España. Portugal, Costa Rica, México, Panamá, Colombia, Chile, Ecuador, Brasil, Perú, Argentina, Venezuela, Cuba.

Una de las iniciativas ha sido la denominada acción "un año de webinars de HCI en Iberoamérica", que comenzó en el 2019, como estrategia para fomentar el conocimiento sobre diversos temas de HCI, los cuales son presentados por reconocidos investigadores y empresarios Iberoamericanos. En estos webinars, los cuales se realizan mensualmente, se invita a dos reconocidos investigadores/empresarios para que cuenten sus experiencias en los temas de HCI. A lo largo del 2019 y 2020, se han organizado 48 webinars, los cuales son libremente (presentaciones y vídeos) distribuidos a toda la comunidad académica, convirtiéndose en una plataforma de Recursos Educativos y Abiertos en temas de HCI (principalmente para la comunidad hispanoparlante) (Granollers et al., 2020). 
De la misma forma, surgió la Red VG-Collab (Red Colaborativa de Investigación y Desarrollo de Videojuegos en Iberoamérica), igualmente auspiciada por la AUIP de España. Esta red tiene como objetivo principal el poder crear una red de académicos, investigadores y profesionales con el objetivo de impulsar actividades conjuntas para la investigación y la transferencia de conocimiento en el ámbito de la mejora y el desarrollo de la industria de videojuegos. Son sintetizados en la Tabla 9 los principales retos y desafíos de investigación de esas dos redes, HCI-Collab y VG-Collab.

Tabla 9. Principales objetivos de investigación de HCI-Collab y VG-Collab

\begin{tabular}{|c|c|}
\hline \multirow[b]{2}{*}{ HCI-Collab } & Objetivos de investigación \\
\hline & $\begin{array}{l}\text { - Hacer un estudio de diversos programas académicos a nivel iberoamericano, también } \\
\text { en USA y Europa en temas de HCI y sus aplicaciones, lo que incluye a la Educación. } \\
\text { - Adecuar el marco conceptual de HCI a las políticas definidas por organismos } \\
\text { internacionales. } \\
\text { - Fomentar investigaciones en ámbito iberoamericano sobre nuevas técnicas de } \\
\text { interacción, lo que incluye realidad mixta e interfaces naturales, y sus implicaciones } \\
\text { en distintas áreas, como Salud, Industria y Educación. } \\
\text { Establecer sinergias con redes de Tecnología Educativa de manera a investigar } \\
\text { elementos de usabilidad y accesibilidad en sistemas computacionales educativos. }\end{array}$ \\
\hline \multirow[t]{2}{*}{ VG-Collab } & Objetivos de investigación \\
\hline & $\begin{array}{l}\text { - Revisar los procesos de desarrollo existentes que apoyan la producción de } \\
\text { videojuegos a nivel Iberoamericano y definir un proceso de desarrollo multicultural } \\
\text { que pueda ser utilizado por empresas y por grupos de investigación en la industria de } \\
\text { software enfocada en videojuegos. } \\
\text { - Establecer sinergias con redes de Tecnología Educativa de manera a investigar la } \\
\text { aplicabilidad e impactos de videojuegos serios y estrategias da gamificación en el } \\
\text { ámbito educativo. }\end{array}$ \\
\hline
\end{tabular}

Una parte de integrantes de la red HCI-Collab proviene de la comunidad LACLO, con la cual la red busca estrechar los lazos de investigación, considerando las muchas intersecciones entre los campos de Tecnologías Educativas e Interacción Humano-Computador. En cuanto a la red VG-Collab, se buscan investigaciones en temas integradores con la comunidad LACLO, como videojuegos educativos y gamificación de la educación.

\subsection{LALA}

LALA (Learning Analytics Latin America) es un proyecto financiado por la Unión Europea dentro de la convocatoria de programas ERASMUS+ que responde a la necesidad de construcción de capacidades para mejorar la gestión y el funcionamiento de las instituciones de educación superior en Latinoamérica (Maldonado-Mahauad et al., 2018). Específicamente, este proyecto busca desarrollar la capacidad local en las Instituciones de Educación Superior (IES) de LatAm para diseñar e implementar herramientas analíticas de aprendizaje para crear y mejorar "procesos y mecanismos de aseguramiento de la calidad". El principal impacto esperado del proyecto es la creación de capacidad local para crear, adaptar, implementar y adoptar herramientas de análisis del aprendizaje para mejorar el proceso de toma de decisiones académicas en las IES de América Latina.

Para poder desarrollar la capacidad local para crear, adaptar y utilizar herramientas de Learning Analytics en América Latina, se han definido cuatro hitos importantes para lograr el objetivo principal. Estos son: (1) Un marco que describa los aspectos metodológicos, técnicos, institucionales, éticos y comunitarios del despliegue de Learning Analytics en el contexto de las IES de América Latina; (2) La adaptación de dos herramientas existentes creadas originalmente en el contexto de Europa al contexto de LatAm. Uno estará dirigido a administradores 
académicos y el otro, a profesores y orientadores. Ambas herramientas se pondrán a prueba para probar su eficacia y mejorar los procesos de toma de decisiones académicas; (3) El Manual de LALA, una guía que contiene el marco metodológico, la recomendación de infraestructura y las experiencias de adopción y mejores prácticas obtenidas durante el piloto. Este será el recurso de orientación para otra IES latinoamericana interesada en adoptar Learning Analytics para modernizar sus operaciones y (4) La Comunidad LALA, que tiene por objetivo continuar los esfuerzos del proyecto, para difundir y explotar sus resultados.

Actualmente la comunidad LALA cuenta con 270 investigadores registrados, 74\% pertenecen a universidades publicas, $22 \%$ a instituciones privadas y $4 \%$ a otro tipo de instituciones como por ejemplo de gobierno. Los investigadores pertenecen a 26 países como son: Argentina, Costa Rica, Granada, Jamaica, Perú, Venezuela, Bolivia, Cuba, Guatemala, México, Puerto Rico, Brasil, Ecuador, Guayana, Nicaragua, Republica Dominicana, Chile, El Salvador, Haití, Paraguay, Surinam, Colombia, Guayana Francesa, Honduras, Panamá y Uruguay. En relación con las IES, actualmente la comunidad LALA ha adherido a 81 instituciones, quienes han manifestado su interés por ser parte de esta red de cooperación. La Tabla 10 se resumen los objetivos de investigación de esa red.

Tabla 10. Principales objetivos de investigación de LALA

\begin{tabular}{|l|l|}
\hline \multirow{4}{*}{ LALA } & Objetivos de investigación \\
\cline { 2 - 3 } & $\begin{array}{l}\text { Mejorar la calidad, eficiencia y relevancia de la Educación Superior en América } \\
\text { Latina, desarrollando capacidades locales para crear, adaptar, implementar y adoptar } \\
\text { herramientas de Learning Analytics que mejoren el proceso de toma de decisiones } \\
\text { académicas. } \\
\text { Desarrollar un framework que describa los aspectos metodológicos, técnicos, } \\
\text { institucionales, éticos y comunales para la implantación de Learning Analytics, en el } \\
\text { contexto de las Instituciones de Educación Superior en América Latina. }\end{array}$ \\
\hline
\end{tabular}

Como parte de las iniciativas de la Comunidad, se han organizado 3 Conferencias Latinoamericanas de Analíticas de Aprendizaje y se han desarrollado dos escuelas de verano ofreciéndose 4 tutoriales y algunos webinars de interés durante el último año. Además, durante el período 2019-2020 se realizaron distintos tipos de visitas a IES en Latinoamérica, quienes solicitaron expresamente colaboración para localizar e invitar expertos en el área, con el propósito de asesorar en la adaptación de herramientas de analítica de aprendizaje. También con el propósito de brindar talleres de capacitación a sus investigadores y profesores, y participar como keynotes en varios eventos de la Región. Finalmente, un punto a destacar es la conformación del grupo Learning Analytics Perú, con quienes se ha podido lanzar el primer programa internacional online de Learning Analytics en convenio con el Instituto para la Calidad de la Pontificia Universidad Católica del Perú. Junto a la comunidad LACLO, esta red está organizando la conferencia conjunta LACLO+LALA en Arequipa, Perú, en 2021.

\section{Retos y desafíos de investigación}

A continuación, como resultado de:

1) las temáticas más frecuentes en la conferencia LACLO en el período 2010-2020, presentado en el ítem 2.2; y

2) las síntesis de retos de investigación expresados al final de cada proyecto o red conectada a LACLO, y presentados entre los ítems 3.1 a 3.9. 
Se pueden extraer grandes temáticas de investigación en Tecnologías Educativas y los desafíos que de ellos provienen, las cuales se resumen en una propuesta de cuatro ejes principales para Latinoamérica, Esta propuesta está sintetizada en la Figura 6.

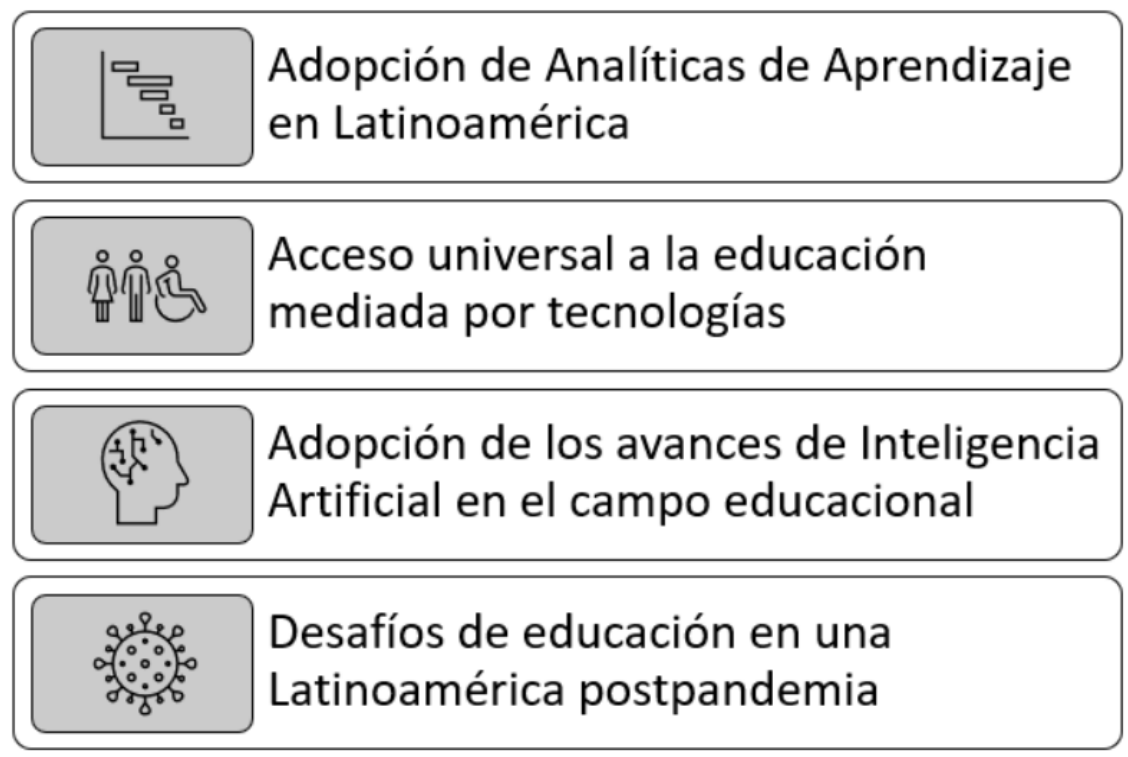

Figura 6. Cuatro grandes ejes de investigación en Tecnologías Educativas para Latinoamérica

Hay que subrayar que esta discusión emerge, como fue mencionado, de un análisis de los temas frecuentes presentado en la conferencia LACLO en el período de 2010-2020 (con énfasis en los últimos años) y de los problemas estudiados en los proyectos y redes de investigación conectados a LACLO. Aunque esta propuesta de grandes ejes no sea exhaustiva, puede arrojar luz a nuevas colaboraciones entre grupos de investigación en Latinoamérica. Sobre cada uno de los cuatro ejes, se presentará una discusión sobre los principales desafíos y retos involucrados en las secciones siguientes.

\subsection{Adopción de Analíticas de Aprendizaje en Latinoamérica}

La teoría de Analítica de Información tiene el propósito de facilitar información objetiva y precisa para soportar la toma de decisiones estratégicas, esto con el fin de mantener la competitividad de las organizaciones, mediante la aplicación de modelos y métodos predictivos que van más allá de simples estadísticas descriptivas. Para las universidades no es ajena la urgencia de contar con información precisa y efectiva para dar soporte a la toma de decisiones estratégicas, con miras a mejorar los procesos y responder a modelos de acreditación nacionales o internacionales. Los procesos de gestión académica generalmente toman más del $80 \%$ del tiempo productivo de los académicos responsables de unidades o programas de formación universitarios. Además, estos procesos de gestión académica se conocen, a partir de reuniones y conversaciones informales con criterio experto, que son repetitivos y atentan fuertemente contra la producción académica y la investigación (Chacón-Rivas et al, 2017).

Por otro lado, la analítica de la información que ha sido más recientemente empleada en los procesos de aprendizaje se centra en mejorar el proceso de aprendizaje mediante el estudio y análisis de los datos producidos durante el proceso en sí. Abarca la recopilación, medición, análisis, presentación de informes y descubrimiento de conocimiento, a partir de datos de estudiantes, profesores e instituciones. (Lemos et al, 2017) (Cechinel, 2020). Claramente uno de los retos más importantes se presenta en el uso de analítica con el fin de lograr un proceso de recomendación de actividades y recursos, a partir de las características o estilos de aprendizaje (Chacón, Garita, 2012) (Gamez, et al, 2012) - también hay que mencionar las críticas de 
Kirschner (2017) a todo ese marco teórico - así como en predicción de éxito académico (Buschetto, et al, 2019).

A pesar de los esfuerzos que se han dado en diferentes universidades y centros de investigación latinoamericanos en materia de colaboración, es evidente el reto de mantener esfuerzos conjuntos a más largo plazo, con miras de obtener procesos de acreditación regionales más que nacionales. Tal y como expresan Cechinel, et al. (2020, p. 1), "América Latina debería utilizar esta capacidad local para modernizar sus sistemas educativos a partir del análisis basado en evidencia proporcionado por analítica de aprendizaje”. La aplicación de la analítica se debe dirigir a apoyar a la comunidad de estudiantes brindando retroalimentación de calidad, en el caso de la comunidad docente se debe apoyar para dar mejor soporte a la población estudiantil en riesgo. Mientras que, en una dimensión de gestión universitaria, la analítica debe impactar en procesos de gestión estratégica en intervención de apoyo y medición de cargas académicas, sin dejar de lado la importancia de los procesos de acreditación. Aún y cuando se cuenta con evidencias del impacto de la analítica desde hace varios años en la comunidad LACLO, por ejemplo, en 2015 se presentó el primer workshop en LACLO/CBIE, en Maceió, Brasil, es claro que la investigación en esta área sigue siendo una oportunidad, reto y desafío de las universidades de la región.

En lo que se refiere a temas de investigación asociados, estos incluyen distintos tópicos que van desde la aplicación de técnicas avanzadas de IA, como Deep Learning (Coelho e Silveira 2017) y temas vinculados a la seguridad de datos personales, entre otros. Sin embargo, se destacan como problemas importantes la disponibilidad y calidad de datos educativos, en los que falta estandarización o uniformidad de los datos disponibles, cuando lo están. La comunidad LALA presentada anteriormente, viene trabajando en muchos de estos temas en el contexto latinoamericano.

A pesar de las diferencias entre los países de Latinoamérica en cuanto a cultura y recursos, un rasgo común es la diversidad de los sistemas de educación superior y de los estudiantes en las instituciones (Cobo \& Aguerrebere, 2018; Fischman \& Ott, 2018). Junto con la diversificación de los tipos de instituciones y programas ofrecidos, la accesibilidad creció para todos los estudiantes, pero particularmente para los de los segmentos de ingresos bajos y medios (Ferreyra et al., 2017). Estos estudiantes, que anteriormente estaban infrarrepresentados, constituyen una pieza fundamental para una mayor calidad y equidad de los sistemas de educación superior. Para garantizar programas de alta calidad a todos, las agencias gubernamentales han implementado o mejorado mecanismos de garantía de calidad en toda la región (Lemaitre, 2017). Por ejemplo, Perú y Ecuador han propuesto recientemente nuevas leyes de educación superior, mientras que Chile y Colombia han reformado sus sistemas de garantía de calidad (Ferreyra et al., 2017). Este fuerte énfasis en la garantía de calidad en los últimos años se ha convertido recientemente en una oportunidad para la adopción de AA en universidades de Latinoamérica. En primer lugar, los mecanismos de acreditación de la calidad han resaltado la importancia del rol docente de las universidades, dando mayor relevancia a los criterios de calidad asociados a la mejora de la enseñanza y el aprendizaje. En segundo lugar, las universidades han invertido esfuerzos significativos en evaluar los resultados del aprendizaje para cumplir con las demandas de responsabilidad, además de recopilar información para monitorear el desempeño de los graduados (Fischman \& Ott, 2018). Como consecuencia, la mayoría de las universidades han fortalecido su capacidad institucional para recopilar y analizar datos educativos para responder a las demandas de calidad. Esta nueva capacidad no sólo ha aumentado la disponibilidad de información sobre el progreso de los estudiantes, sino que también ha creado conciencia sobre la importancia de mejorar las prácticas curriculares y docentes basadas en la evidencia educativa. Considerando la cantidad de datos educativos acumulados en las últimas dos décadas, Cobo y 
Aguerrebere (2018) indican que las universidades de Latinoamérica podrían implementar servicios de AA para abordar desafíos que todos los sistemas de educación superior de la región tienen en común, como mejorar la calidad y reducir las tasas de abandono de los estudiantes. Sin embargo, las universidades de Latinoamérica aún necesitan ampliar su capacidad antes de adoptar AA a nivel institucional. Primero, necesitan instalar sistemas de información válidos y confiables para integrar los datos que actualmente se almacenan y administran por separado (Lemaitre, 2017). En segundo lugar, deben comprender las perspectivas de las partes interesadas con respecto a la protección y la privacidad de datos, además de implementar políticas institucionales de acuerdo con los derechos constitucionales o las regulaciones que actualmente se están revisando a la luz del GDPR (Villan, 2019). En tercer lugar, necesitan crear comunidades de práctica para adquirir y generar conocimiento de AA, aprovechando las iniciativas existentes para promover la colaboración y el desarrollo de capacidades hacia la adopción de AA (Maldonado-Mahauad et al., 2018). Debido a estos problemas de capacidad, hay un largo camino para pasar de la investigación a la adopción institucional de los servicios de AA en las universidades de Latinoamérica (Cobo \& Aguerrebere, 2018; Pérez-Sanagustín et al., 2018). Sin embargo, la capacidad de las instituciones para recopilar y analizar datos educativos para cumplir con los sistemas de acreditación de calidad podría sentar las bases para la adopción de AA. Según los investigadores, los datos de calidad son un componente esencial del éxito de las iniciativas de AA (Arnold et al., 2014; Greller \& Drachsler, 2012; Tsai et al., 2018) y su disponibilidad crea un punto de partida para diseñar e implementar Servicios de AA.

\subsection{Acceso universal a la educación mediada por tecnologías}

En los últimos cinco años antes de la pandemia del COVID-19 en los países de América Latina, se han logrado ciertos avances al ampliar la cobertura en educación inclusiva y el reconocer la necesidad de lograr la adquisición de competencias en materia de TICs por los estudiantes con alguna discapacidad, considerando su condición socioeconómica y otras dimensiones, como género y etnia, por ejemplo. Debido a que la desigualdad ya existente ha sido exacerbada por la pandemia, las opciones de política que se diseñen para la educación en la actual coyuntura deben reconocer deudas históricas con estos grupos para garantizar su derecho a la educación, tanto en lo referido a la disponibilidad y el acceso a oportunidades de aprendizaje, como a la provisión de una educación de calidad, relevante y adaptada a sus condiciones, necesidades y aspiraciones (Vargas, 2019).

Dado los efectos de la pandemia y la consecuente crisis sanitaria, social y económica es urgente atender en los países de América Latina ha traído consigo el riesgo de desvinculación educativa y abandono escolar de los grupos más vulnerables (Lion, 2019) en particular los estudiantes con algún tipo de vulnerabilidad social, lo que incluye estudiantes con discapacidad, grupos indígenas y/o afrodescendientes en algunos contextos, etc. La combinación de estas condiciones de desigualdad social refuerza los círculos de exclusión y de vulnerabilidad.

Entre los proyectos mencionados anteriormente, el Proyecto SELI busca una disminución de la brecha digital mencionada por Tomczyk (2019) al proponer un ecosistema digital de apoyo a la educación inclusiva. En el sentido de mitigar las diferentes barreras de acceso a materiales educativos, proyectos como LATIn, ESVI-AL y RIURE buscaron distintas soluciones para el problema.

El hacer factible el acceso a las tecnologías de la información y comunicación ayuda a mitigar la condición educativa de grupos vulnerables, se pueden citar lo siguiente retos:

- Afianzar con TICs la participación de los padres de familia en apoyar en la educación de sus hijas e hijos tanto dentro y fuera de actividades fuera del hogar 
- Potenciar con las TICs las competencias de profesorado que necesita la sociedad actual en términos de diversidad, equidad y sustentabilidad y su vinculación con la transferencia de esta formación en el sistema educativo (Lion, 2019) .

- Enfoques centrados en las prácticas y en los desafíos en relación con los aprendizajes y habilidades necesarias cuando hay mediación tecnológica en tiempos de grandes volúmenes de información, un auge de la inteligencia artificial y la "subjetividad ampliada" con posibilidades hasta ahora inéditas y aumentadas por procesos cognitivos superiores (Muñoz, 2020a) (Sadin, 2017).

- Hacer uso de las TIC en las respuestas diversas que requieren distintos grupos, incorporando respuestas pedagógicas sensibles y pertinentes a las diferencias culturales, lingüísticas, de género y de accesibilidad (Muñoz, 2020b).

Finalmente, el desarrollo de herramientas educativas realizadas por equipos interdisciplinarios y no sólo por tecnólogos, como promueve LACLO en su comunidad, brinda un nuevo y amplio enfoque del uso de la tecnología, especialmente para desarrollar el Diseño Universal del Aprendizaje (CAST,2020), (Viola et al. 2020).

\subsection{Adopción de los avances de Inteligencia Artificial en el campo educacional}

Se puede considerar que en la década de 2010-2020 sobre la cual se escribe este artículo, uno de los desarrollos más relevantes de la Inteligencia Artificial (IA) aplicados a la educación han sido los Sistemas Recomendadores (Casali et al., 2012), que pueden recomendar recursos de aprendizaje de diferente granularidad, a alumnos de distintos niveles educativos. Dentro de estos sistemas se han aplicado distintas técnicas de recomendación: basadas en contenidos, basadas en conocimientos y con el avance del uso de recursos en distintas plataformas, técnicas de filtrado colaborativo (Lemos et al., 2016). Estas últimas técnicas, como otros modelos de aprendizaje automatizado, han visto muchas veces obstaculizada su aplicación para poder obtener buenos modelos por la falta de disponibilidad de grandes bancos de datos de buena calidad. Esto tiene varios aspectos a considerar: como la falta de uso masivo de recursos de aprendizaje comparada con otros elementos (por ej. compras de productos, música, películas, etc.), la falta de acceso a información de estudiantes debido a políticas de privacidad de la información institucional o a la falta de regulaciones claras. Estos problemas son los que en parte enfrenta IA y han sido tratados en la Sec. 4.1. También se continuó con el avance en los Intelligent Tutoring Systems, desarrollando herramientas de personalización de trayectos educativos. Si bien la investigación y desarrollo de estas aplicaciones ha crecido, no han alcanzado masividad. Una dificultad es la falta de metadatos de los recursos a recomendar y además, la necesidad de contar con la información de mayor cantidad de usuarios para poder utilizar técnicas colaborativas, que aprendan el perfil a partir de la experiencia de un estudiante-usuario y que permitan incorporar también herramientas de HCI para adaptar sus interfaces.

Respecto a la usabilidad de Repositorios de objetos de aprendizaje, como se ha mencionado en la red RIURE (Sec. 3.2) se ha trabajado en herramientas que utilizando la IA ayuden a automatizar distintas tareas para la carga y extracción de información. Hay otras áreas que han recibido el impacto de la IA como, por ejemplo, la conformación automática de grupos de acuerdo a los perfiles de los alumnos, herramientas de evaluación colaborativa, personalización de las interfases (Sec. 3.6), analítica de aprendizaje (Sec. 3.7), de modo de poder realizar mejor tanto la tarea docente, como la gestión del sistema educativo. Por otra parte, muchos desafíos de IA en educación están ligados a los avances de otras áreas como Procesamiento de Lenguaje Natural (PLN o NLP - Natural Language Processing) y Visión Computacional, de modo de poder manipular de forma automática texto no estructurado y material multimedia. Recientemente, ambas áreas han desarrollado un crecimiento expresivo a 
través de las herramientas de Deep Learning y se espera que sus aplicaciones impacten al dominio de la educación.

A partir del 2020, la pandemia COVID-19 ha obligado a trasladar los cursos presenciales a una modalidad virtual de una forma vertiginosa, utilizando masivamente plataformas virtuales y distintos recursos en línea. Esto ha generado una gran cantidad de recursos educativos abiertos y muchísimas interacciones de los usuarios (docentes y alumnos) con las distintas plataformas. Por lo cual ahora hay una gran cantidad de datos e información para ser explorada por herramientas de IA, tanto para poder mejorar la selección y recomendación de recursos, así como también, para dar mejor soporte a las estrategias y gestión educativa.

Sigue estando en la agenda de las aplicaciones de la IA en educación los siguientes desafíos: la personalización de la educación utilizando modelos de aprendizaje más complejos; brindar herramientas para producir mejores contenidos y mantenerlos actualizados; apoyar la automatización de tareas administrativas y garantizar la accesibilidad a la educación a todas las personas.

Por otra parte, ante los grandes avances de la IA en estos últimos años es necesario que todos los países y especialmente en la región Latinoamericana, se puedan establecer planes estratégicos para tener soberanía tecnológica en esta área. Otro aspecto sumamente relevante es la educación ciudadana sobre las bases y peligros que pueden presentar estos desarrollos, para que todos podamos beneficiarnos de estas tecnologías, pero a la vez, ser ciudadanos críticos y cuidadosos. En particular, respecto a la privacidad y protección de datos, este es un derecho fundamental de toda persona a tener el control de aquellos datos sensibles. Con el uso masivo de las TICs, se está cada vez más expuesto a un uso no adecuado de la información personal. Es por esta razón, que se necesitan regulaciones que protejan nuestros datos y lograr empoderar a los ciudadanos, brindándoles las competencias mediáticas necesarias para acceder, comprender, analizar, el impacto de las TIC. Se debate la didáctica de su enseñanza en la escolarización obligatoria, incluyendo pensamiento computacional y la programación, introduciendo algunos contenidos de IA, de modo de brindar una formación que permita utilizar de forma inteligente y crítica estas aplicaciones que nos sorprenden día a día. En tercer lugar, hay que establecer principios, políticas y leyes para el uso responsable de IA. Los distintos gobiernos y foros mundiales están discutiendo las cuestiones éticas y regulaciones que deben establecerse al respecto. Incluyen directrices éticas como: respeto a la autonomía humana, prevención del daño, equidad y explicabilidad (UE, 2018) y deberían seguir los valores de ser: imparciales, confiables, inclusivos, transparentes, responsables y asegurar la privacidad y seguridad de la información (Microsoft, 2018). Dentro de las técnicas de IA hay mucho potencial para desarrollar aplicaciones que colaboren en los distintos procesos educativos, mejorando su calidad, accesibilidad y equidad, pero deben desarrollarse respetando estas directrices éticas.

\subsection{Desafíos de educación en una Latinoamérica postpandemia}

Según Waissbluth (2019, p. 177). cuatro objetivos comunes fueron establecidos por la Unión Europea hasta el año de 2020 con vistas a solucionar o mitigar los problemas educativos:

- Hacer del aprendizaje permanente y de la movilidad una realidad;

- Mejorar la calidad y eficacia de la educación y la formación;

- Fomentar la igualdad, la cohesión social y la ciudadanía activa;

- Incrementar la creatividad y la innovación, incluido el espíritu empresarial, en todos los niveles de la educación y la formación. 
De la misma manera, la UNESCO (2020) establece ciertas recomendaciones para mitigar la crisis educativa debida al COVID 19, entre las cuales señalan:

- Proteger el financiamiento de la educación y coordinar el impacto

- Construir sistemas educativos resilientes para un desarrollo equitativo y sostenible

- Re imaginar la educación y acelerar el cambio en la enseñanza y el aprendizaje

Es necesario invertir en investigaciones que busquen soluciones tecnológicas para los estudiantes en situación de vulnerabilidad social, pero también para los profesores, como subrayan Araujo et al. (2020) y todos los demás involucrados, como las familias y todo el contexto de la comunidad académica.

Mientras tanto, los posibles escenarios educativos postpandemia todavía no quedan claros al momento de la escritura de este artículo. Aunque Kuklinski y Cobo (2020) presenten reflexiones firmes sobre los modelos de educación híbrida, se deben realizar investigaciones profundas sobre la democratización de las soluciones que se van a proponer, de manera de no crear toda una generación de excluidos de los sistemas educativos por su exclusión digital. Esto es sumamente importante en contextos de alta inequidad social, como es Latinoamérica (Tomczyk et al., 2019).

\section{Conclusiones y Trabajos Futuros}

En la última década, hemos sido testigos del aumento de la colaboración en investigación en la región Latinoamericana, en el tema de tecnologías educativa; aumentando la presencia de investigadores, la vinculación de grupos de investigación, la creación de redes de colaboración, así como el incremento de jornadas y eventos, que han servido de encuentro y formación de nuevos cuadros, que han funcionado como articuladores en la estructuración de proyectos, publicaciones y formación de investigadores en el área. La colaboración en redes es un común denominador y la conformación de la comunidad LACLO ha constituido un movimiento que, en forma espiralada y creciente ha evolucionado, incorporando nuevos actores, sumando nuevos países y temas de investigación, brindando aportes significativos en el área de tecnologías de aprendizaje, así como asociándose a redes ya existentes y al mismo tiempo estimulando nuevas redes de investigación.

LACLO comenzó como una pequeña comunidad interesada en investigar e innovar en aspectos relacionados a los OA y sus repositorios, pero en poco tiempo fue incorporando nuevos temas de interés, como los MOOCs, Aprendizaje móvil, Televisión educativa y Aprendizaje ubicuo, Analíticas de aprendizaje y Minería de datos educativos, Aula invertida, Videojuegos, Realidad Virtual y Aumentada, Aprendizaje personalizado, Adaptabilidad y Accesibilidad. En este sentido, importantes grupos temáticos conviven en estas tendencias, conformando equipos de trabajo, incorporando líneas de investigación y proponiendo nuevos proyectos, que puedan ser puntas de lanza en Latinoamérica y referentes para Europa y el resto del mundo.

Este artículo presenta una propuesta de cuatro grandes ejes de investigación en el área de tecnologías Educativas en Latinoamérica que, aunque no sea un listado exhaustivo, puede servir como guía para futuros proyectos, redes y propuestas de investigación. Es sabido que Latinoamérica presenta un plantel muy variado de realidades educativas que no se pretende aquí reducirlas a una simplificación por medio de una organización temática - al revés, los ejes pretenden servir como organizadores de futuras colaboraciones en investigación, mezclando áreas de interés de los grupos de investigación transnacionales y las demandas educativas intrínsecas a cada región. 
Hay muchos desafíos para el futuro en lo que se refiere a las posibilidades de sinergia de investigación en Tecnologías Educativas en Latinoamérica. Nuestras diferencias son altamente sobrepasadas por todo lo que nos une, que son las necesidades de pueblos que han sufrido procesos colonizadores por siglos, que han construido distintas Historias que mantienen innumerables intersecciones y que nos traen a un único tiempo presente. Que las redes de investigación y los proyectos que han sido mencionados en este artículo puedan ser inspiradores para las nuevas redes y proyectos en un futuro que, si bien es incierto, está también lleno de posibilidades.

\section{Referencias}

Araujo, R. M., Amato, C. A. de la H., Martins, V. F., Eliseo, M. A., \& Silveira, I. F. (2020). COVID-19, Mudanças em Práticas Educacionais e a Percepção de Estresse por Docentes do Ensino Superior no Brasil. Revista Brasileira de Informática na Educação, 28, 864-891. doi: 10.5753/rbie.2020.28.0.864 [GS Search]

Bender, C., Deco, C., Casali, A., \& Kantor, R. (2013). Libros Digitales Abiertos: análisis de preferencias y prácticas por parte de profesores y estudiantes de la UNR. Revista TE \& ET, 10, 16-25. Disponible en: http://sedici.unlp.edu.ar/handle/10915/27722 [GS Search]

Buenaño-Fernandez, D., Villegas-Ch, W., \&Lujan-Mora, S. (2018). Using text mining to evaluate student interaction in virtual learning environments. IEEE World Engineering Education Conference (EDUNINE), 1-6. doi: 10.1109/EDUNINE.2018.8450969 [GS Search]

Buschetto Macarini, L. A. Cechinel, C., Batista Machado, M. F., Culmant Ramos, V. F., \& Munoz, R. (2019). Predicting Students Success in Blended Learning-Evaluating Different Interactions Inside Learning Management Systems. Applied Sciences, 9(24), 5523. doi: $\underline{10.3390 / a p p 9245523}$ [GS Search]

Casali, A., Deco, C., Bender, C., \& Gerling, V. (2012). Recommender System for Personalized Retrieval of Learning Objects. En O. C. Santos y J. G. Boticario (Eds.), Educational Recommender Systems and Technologies. Practices and Challenges (182-210), Madrid, IGI Global. doi: 10.4018/978-1-61350-489-5.

Casali, A., Deco, C., \& Beltramone, S. (2016). An Assistant to Populate Repositories: Gathering Educational Digital Objects and Metadata Extraction. IEEE Journal of Latin-American Learning Technologies, 11(2), 1-8. doi: 10.1109/RITA.2016.2554018 [GS Search]

Casali, A., Cechinel, C., \& Ochoa, X. (2015). Edición Especial: Estrategias para Mejorar la Usabilidad de Repositorios Educativos. VAEP-RITA, 3(2), 76-77. Disponible en: http://rita.det.uvigo.es/VAEPRITA/index.php?content=Num_Pub\&idiom=Es\&visualiza=1\&vol $\underline{\text { umen }=3 \& \text { numero }=2 \& \text { orden }=\text { desc }}$

Casali, A., Bender, C., Deco, C., \& Fontanarrosa, S. (2014). Extracción Automática de Metadatos como Soporte para el Autoarchivo de Objetos Digitales en Repositorios. Revista Colombiana de Computación, 15(2), 135-160. doi: 10.29375/25392115.2490 [GS Search]

Casali, A., Deco, C., Romano, A., \& Tomé, G. (2013). An assistant for loading Learning Object Metadata: An ontology-based approach. Interdisciplinary Journal of E-Learning and Learning Objects, IJELLO, 9, 77-87. Disponible en: http://www.ijello.org/Volume9/IJELLOv9p077087CasaliFT81.pdf [GS Search]

Casali, A., Silva Sprock, A., Alves, C., Deco, C., Silveira, I. F., Muñoz-Arteaga, J., Gomes dos Santos, J., Broisin, J., Morales, R., C Hernández, Y., \& Ochoa, X. (2012). Collaborative Methodologies for Writing Open Educational Textbooks: a State-of art Review. Anais dos Workshops do Congresso Brasileiro de Informática na Educação, Rio de Janeiro, Brasil. [GS 


\section{$\underline{\text { Search }]}$}

CAST. (2020). UDL Guidelines. Disponible en: http://www.cast.org/

Chacon-Rivas, M, Francesa-Alfaro, A., Carreno-Leon, M., \& Sandoval-Bringas, A. (2017). Integrating academic analytics for supporting accreditation and international cooperation: TEC and UABCS experience. Twelfth Latin American Conference on Learning Technologies (LACLO), La Plata: IEEE, 1-7. doi: 10.1109/LACLO.2017.8120888 [GS Search]

Chacon-Rivas, M., \& Garita, C. (2014). A Successful OSS Adaptation and Integration in an ELearning Platform: TEC Digital. En Luis Corral et al. (Eds), Open Source Software: Mobile Open Source Technologies, IFIP Advances in Information and Communication Technology, Berlin, Heidelberg: Springer, 143-46. doi: 10.1007/978-3-642-55128-4 19 [GS Search]

Chacón-Rivas, M., \& Garita, C. (2012). TEC Digital: A Case Study of an e-Learning Environment for Higher Education in Costa Rica. International Conference on Information Technology Based Higher Education and Training (ITHET), 1-6. doi: 10.1109/ITHET.2012.6246061 [GS Search]

Cechinel, C., Diaz, A. V., Avila, C., Motz, R., Mendez, E., García, M., \& Diaz, P. (2015). Una experiencia de un curso online sobre Recursos Educativos Abiertos en el Marco del Programa de Apoyo al Sector Educativo del Mercosur. Anais temporários do LACLO 2015, 286-295. Disponible en: https://www.br-ie.org/pub/index.php/teste/article/view/5810 [GS Search]

Cechinel, C. (2013). Scientific Collaboration between Countries in LACLO from a Social Network Analysis Perspective. Actas de la Octava Conferencia Latino-Americana de Objetos y Tecnologías de Aprendizaje, Valdivia, Chile.

Cechinel, C., Camargo, S. S., Sicilia, M. A. \& Sanchez-Alonso, S. (2016). Mining Models for Automated Quality Assessment of Learning Objects. Journal of Universal Computer Science 22, 94-113. doi: 10.3217/jucs-022-01-0094 [GS Search]

Cechinel, C., Ochoa, X., Lemos dos Santos, H., Carvalho Nunes, J., Rodés, V., \& Marques Queiroga, E. (2020). Mapping Learning Analytics Initiatives in Latin America. British Journal of Educational Technology, 51(4), 892-914. doi: 10.1111/bjet.12941 [GS Search]

Cervantes, O., Motz, R., Castillo, E., \&Velázquez, J. (2018). Uso de métricas sociales para descubrir patrones de interacción que impactan el aprendizaje. Proceedings of the 1st Latin American Workshop on Learning Analytics, Guayaquil, Ecuador. CEUR Workshop Vol-2231. Disponible en: http://ceur-ws.org/Vol-2231/LALA_2018_paper_18.pdf

Cobo, C., \& Aguerrebere, C. (2018). Building capacity for learning analytics in Latin America. In C. Ping Lim y V. L. Tinio (Eds.), Learning Analytics for the Global South (pp. 63-67). Quezon City, Philippines: Foundation for Information Technology Education and Development, Inc. Disponible en: http://digital.fundacionceibal.edu.uy:8080/jspui/bitstream/123456789/243/2/LearningAnalytics-Full-Paper-2.pdf\#page=63 [GS Search]

Coelho, O. B., \& Silveira, I. F. (2017). Deep learning applied to learning analytics and educational data mining: A systematic literature review. Simpósio Brasileiro de Informática na EducaçãoSBIE, 28(1), 143-152. doi: 10.5753/cbie.sbie.2017.143 [GS Search]

Comisión de Expertos Unión Europea (2018) Directrices Éticas para una IA confiable, Disponible en: https://ia-latam.com/portfolio/pautas-eticas-para-una-ia-confiable-comunidad-europea/UE

Deco, C., Bender, C., Casali, A., \& Kantor, R. (2013). Creación de libros abiertos en América Latina: metodología, plataforma y estrategias. Actas del XV Workshop de Investigadores en Ciencias de la Computación. Entre Ríos, Argentina. Disponible en: http://biblioteca.puntoedu.edu.ar/handle/2133/3296 [GS Search]

Deco, C., Bender, C., Casali, A., \& Motz, R. (2017). Analyzing the evolution of learning technology 
area using science mapping. Proceedings of the Twelfth Latin American Conference on Learning Technologies (LACLO), La Plata, Argentina, 1-8. doi: $\underline{\text { 10.1109/LACLO.2017.8120930 [GS Search] }}$

Díaz, A., Fernández, A., Motz, R., Cechinel, C., Garcia Diaz, M. E., \& Mendez, H. (2014). REMAR: REd Mercosur para la accesibilidad y la generación colaborativa de recursos educativos abiertos. IX Congreso sobre Tecnología en Educación \& Educación en Tecnología, Argentina, La Rioja. Disponible en: https://digital.cic.gba.gob.ar/handle/11746/3187 [GS $\underline{\text { Search }}$

Ferreyra, M. M., Avitabile, C., Botero Álvarez, J., Haimovich Paz, F., \& Urzúa, S. (2017). At a Crossroads: Higher Education in Latin America and the Caribbean. Washington DC: World Bank. doi: 10.1596/978-1-4648-0971-2 [GS Search]

Fischman, G. E., \& Ott, M. (2018). Access, equity and quality trends in Latin America's public universities. International Journal of Educational Development, 58, 86-94. doi: 10.1016/j.ijedudev.2016.11.002 [GS Search]

Gámez, I., Garita, C., \& Chacón-Rivas, M. (2012). Generación de Sugerencias de Rutas de Aprendizaje Adaptativas en Entornos de e-learning. En Conferencia Latinoamericana en Informática-CLEI. 2012. Medellín, Colombia: IEEE, 1-10.

Granollers, T., Muñoz, J., Collazos, C., \& Luna, H. (2020). A year of webinars in Latin America. Interactions, 27(6), 62-65. doi: 10.1145/3424684

Kirschner, P. A. (2017). Stop propagating the learning styles myth. Computers \& Education, 106, 166-171. doi: 10.1016/j.compedu.2016.12.006 [GS Search]

Kuklinski, H. P., \& Cobo, C. (2020). Expandir la universidad más allá de la enseñanza remota de emergencia. Ideas hacia un modelo híbrido post-pandemia. Barcelona: Outliers School, 2020. Disponible en: https://outliersschool.net/wpcontent/uploads/2020/05/Expandir_la_universidad.pdf

Lemaitre, M. J. (2017). Quality assurance in Latin America: current situation and future challenges. Tuning Journal for Higher Education, 5(1), 21-40. doi: 10.18543/tjhe-5(1)-2017pp21-40 [GS Search]

Lemos, H. S., Cechinel, C., \& Matsumura, R. A. (2016). A Comparison among Approaches for Recommending Learning Objects through Collaborative Filtering Algorithms. Program: electronic library and information systems, 51(1), 35-51. doi: 10.1108/PROG-05-2016-0044

Lemos dos Santos, H., Cechinel, C., Nunes, J. B. C., \& Ochoa, X. (2017). An initial review of learning analytics in Latin America. 2017 Twelfth Latin American Conference on Learning Technologies (LACLO), 1-9, Argentina, La Plata. doi: 10.1109/LACLO.2017.8120913 [GS Search]

Lion, C. (2019). Los desafíos y oportunidades de incluir tecnologías en las prácticas educativas. Análisis de casos inspiradores, IIPE-UNESCO Buenos Aires oficina para América Latina.

Macarini, L. A., Cechinel, C., Santos, H. L. D., Ochoa, X., Rodés, V., Alonso, G. E., \& Díaz, P. (2019). Challenges on implementing learning analytics over countrywide K-12 data. En actas de 9th international conference on learning analytics \& knowledge, LAKS19, (pp. 441-445). doi: $\underline{10.1145 / 3303772.3303819}$ [GS Search]

Macarini, L. A., Lemos dos Santos, H., Cechinel, C., Ochoa, X., Rodés, V., Pérez Casas, A., \& Díaz, P. (2020). Towards the implementation of a countrywide K-12 learning analytics initiative in Uruguay. Interactive Learning Environments, 28(2), 166-190. doi: $\underline{10.1080 / 10494820.2019 .1636082}$ [GS Search]

Maldonado-Mahauad, J., Hilliger, I., De Laet, T., Millecamp, M., Verbert, K., Ochoa, X., \& Pérez1088 
Sanagustín, M. (2018). The LALA project: Building capacity to use learning analytics to improve higher education in Latin America. En actas de 8th international learning analytics \& knowledge conference (pp. 630-637). Disponible en: https://www.lalaproject.org/wpcontent/uploads/2019/04/LALA framework English.pdf [GS Search]

Martins, V., Oyelere, S. S., Tomczyk, L., Barros, G., Akyar, O., Eliseo, M. A., Amato, C., \& Silveira, I. F. (2019). A blockchain microsites-based ecosystem for learning and inclusion. Brazilian Symposium on Computers in Education (Simpósio Brasileiro de Informática na Educação-SBIE), 30(1), 229-238. doi: 10.5753/cbie.sbie.2019.229 [GS Search]

Microsoft (Smith B. Shum H. Eds.) (2018). El Futuro Computarizado: La Inteligencia Artificial y su rol en la sociedad, Microsoft.

Motz, R., Cervantes, O., \& Echenique, P. (2018). Sentiments in Social Context of Student Modelling. En actas de XIII Latin American Conference on Learning Technologies (LACLO) (pp. 484-491). doi: 10.1109/LACLO.2018.00086 [GS Search]

Muñoz Arteaga, J. (2020a) Collaborative Production Model of Educative Resources for HumanComputer Interaction Community in Latino America. En: Yves Rybarczyk (Ed.), Human 4.0 From Biology to Cybernetic, Editor, Editorial IntechOpen. doi: 10.5772/intechopen.90992

Muñoz Arteaga, J. (2020b). Educational Applications as a support for Reading Disability at Elementary School. (040719-011846), En: Ricardo Mendoza-González, Huizilopoztli LunaGarcia, Alfredo Mendoza-González (Eds.), UXD and UCD Approaches for Accessible Education, 127-152, IGI-GLOBAL. doi: 10.4018/978-1-7998-2325-4.ch008 [GS Search]

Ochoa, X., Silveira, I. F., \& Sprock, A. S. (2011). Collaborative open textbooks for Latin AmericaThe LATIn project. En: Actas de International Conference on Information Society (i-Society 2011) (pp.398-403). IEEE. doi: 10.1109/i-Society18435.2011.5978479 [GS Search]

Ochoa, X., Casali, A., Deco, C., Gerling, V., Silveira, I. F., Fager, J., Carrillo, G., Parra, G., MuñozArteaga, J., Maturana, J., Araya, E., \& Motz, R. (2013). Analysis of existing technological platforms for the collaborative production of Open Textbooks. En EdMedia+ Innovate Learning (p. 1106-1115). Association for the Advancement of Computing in Education (AACE). Disponible en: https://www.learntechlib.org/p/112098/ [GS Search]

Pérez-Sanagustín, M., Hilliger, I., Maldonado-Mahauad, J., Pérez-álvarez, R., Ramírez, L., MuñozMerino, P. J., Whitelock-Wainright, A. (2018). LALA Framework. Disponible en: https://www.lalaproject.org/

Rodés, V., Casas, A. P., Ochoa, X., \& Silveira, I. F. (2012). Percepciones, actitudes y prácticas respecto a los libros de texto, digitales y en formatos abiertos por parte de estudiantes de universidades de América Latina. Anais dos Workshops do Congresso Brasileiro de Informática na Educação. [GS Search]

Rodés, V., Rodríguez, C., Maturana, J., Hernández, Y., Cuadros Vargas, E., \& Podetti, M. (2013). Strategies for Implementing the Adoption of Open Textbooks Initiatives: State of the Art Review. In: International Journal of e-Education, e-Business, eManagement and e-Learning (IJEEEE 2013), 3(1), 51-56. doi: 10.7763/IJEEEE.2013.V3.192 [GS Search]

Rodes-Paragarino V., Gewerc Barujel A., \& Llamas-Nistal M. (2015). Use of Repositories of Digital Educational Resources: State-of-the-Art Review. IEEE Revista Iberoamericana de Tecnologías del Aprendizaje, 11(2), 73-78. doi: 10.1109/RITA.2016.2554000 [GS Search]

Rodés Paragarino, V., Pelerino Francolino, Flavio, F., Gewerc Barujel, Adriana, A., \& Llamas Nistal, M. (2016). A characterization of users of digital educational resources repositories the case of the Latin American Community of Learning Objects. En XI Latin American Conference on Learning Objects and Technology (LACLO), 1-8. doi: 10.1109/LACLO.2016.7751793 [GS $\underline{\text { Search] }}$ 
Sadin, W. (2017). La humanidad aumentada. La administración digital del mundo. Buenos Aires: Caja Negra.

Silveira, I. F., Ochoa, X., Silva Sprock, A., Notargiacomo, P., \& Hernández, Y. (2012). Towards New Computational Architectures for Mass-Collaborative Open Educational Resources. En: International Journal of Digital Information and Wireless Communications 1(2), 526-540. Disponible en: http://sdiwc.net/digital-library/towards-new-computational-architectures-formasscollaborative-openeducational-resources [GS Search]

Silveira, I. F., Ochoa, X., Cuadros-Vargas, A., Pérez Casas, A., Casali, A., Ortega, A., Sprock, A. S., Alves, C. H., Collazos-Ordoñez, C. A., Deco, C., Cuadros-Vargas, E., Knihs, E., Parra, G., Muñoz-Arteaga, J., Santos, J. G., Broisin, J., Omar, N.; Motz, R., Rodés, V., \& Bieliuckas, Y. (2013). A digital ecosystem for the collaborative production of open textbooks: The LATIn methodology. En: Journal of Information Technology Education: Research, 12, 225-249. Disponible en: http://www.jite.org/documents/Vol12/JITEv12ResearchP225246SilveiraFT89.pdf [GS Search]

Silveira, I. F., Mustaro, P., Silva, L., Nizam, O., Knihs, E., Rodés, V, Silva Sprock, A., \& Ochoa, X. (2013). O desafio do acesso e permanência no Ensino Superior frente ao custo dos livros didáticos: proposta de uma arquitetura de criação e disseminação de livros digitais colaborativos abertos. Em: Actas do XXXIII Congresso da Sociedade Brasileira de Computação 2013 (CSBC2013). II Workshop de Desafios da Computação Aplicada à Educação (DEsafIE!2013), Julio 2013. Maceió. Brasil. [GS Search]

Silveira, I. F. (2016). OER and MOOC: The need for openness. Issues in Informing Science \& Information Technology, 13, 209-223. doi: 10.28945/3478 [GS Search]

Tomczyk, Ł., Eliseo, M. A., Costas, V., Sánchez, G., Silveira, I. F, Barros, M. J., AmadoSalvatierra, H. R., \& Oyelere, S. S. (2019). Digital Divide in Latin America and Europe: Main characteristics in selected countries. En 14th Iberian Conference on Information Systems and Technologies (CISTI), 1-6. doi: 10.23919/CISTI.2019.8760821 [GS Search]

Tomczyk, Ł., Martins, V. F., Eliseo, M. A., Silveira, I. F., de la Higuera Amato, C., \& Stošić, L. (2020). ICT and education in Brazil - NGO, local government administration, business and higher education expert perspective. World Journal on Educational Technology: Current Issues, 12(4), 401-424. doi: 10.18844/wjet.v12i4.5198 [GS Search]

UNESCO (2020). Policy Brief: Education during COVID-19 and beyond, August 2020. Disponible en: https://www.un.org/development/desa/dspd/wpcontent/uploads/sites/22/2020/08/sg_policy_brief_covid-19_and_education_august_2020.pdf

Vargas, C. (2019). Leaving no one behind: bringing equity and inclusion back into education. En Tett L. y Hamilton M. (Eds.), Resisting Neoliberalism in Education: Local, National and Transnational Perspectives, Bristol, Policy Press. doi: 10.1332/policypress/9781447350057.003.0017 [GS Search]

Viola Deambrosi, M.., Motz, R., \& Eliseo, M. A. (2020). Why Universal Design for Learning need an Ontology?. 15th Iberian Conference on Information Systems and Technologies (CISTI), Sevilla, Spain, 1-6. doi: 10.23919/CISTI49556.2020.9141109

Waissbluth, M. (2019). Educación para el siglo XXI: El desafío latinoamericano. Santiago, Chile: Fondo de Cultura Económica, Salesianos Impresores S.A. doi: 10.29393/Pa66-21WMPF10021 